\title{
Finite-time dissipative synchronization of discrete- time semi-Markovian jump complex dynamical networks with actuator faults
}

\author{
N. Sakthivel
}

Bharathiar University

S. Pallavi

Bharathiar University

Yong-Ki Ma ( $\square$ ykma@kongju.ac.kr)

Kongju National University

V. Vijayakumar

Vellore Institute of Technology: VIT University

\section{Research Article}

Keywords: Finite-time synchronization, Complex dynamical networks, Dissipativity, Semi-Markovian jumping, Reliable control.

Posted Date: February 16th, 2022

DOl: https://doi.org/10.21203/rs.3.rs-899072/v1

License: (9) This work is licensed under a Creative Commons Attribution 4.0 International License. Read Full License 


\title{
Finite-time dissipative synchronization of discrete-time semi-Markovian jump complex dynamical networks with actuator faults
}

\author{
N.Sakthivel ${ }^{1}$, S.Pallavi ${ }^{1}$, Yong-Ki Ma ${ }^{2 *}$, and V.Vijayakumar ${ }^{3}$ \\ 1 Department of Applied Mathematics, Bharathiar University, Coimbatore - 641 046, \\ Tamilnadu, India. \\ 2 Department of Applied Mathematics, Kongju National University, Chungcheongnam-do 32588, \\ Republic of Korea. \\ 3 Department of Mathematics, School of Advanced Sciences, Vellore Institute of Technology, \\ Vellore - 632014, Tamilnadu, India.
}

\begin{abstract}
This paper is concerned with the problem of finite-time synchronization for a class of discretetime semi-Markovian jumping complex dynamical networks(CDNs) with actuator faults based on reliable control. The main aim of this paper is to design a state feedback controller such that the resulting closed-loop system is finite-time synchronized under a prescribed dissipativity performance level even in the presence of actuator failures. Moreover, a stochastic nature followed by Bernoulli distribution is described in the considered networks due to the occurrence of probabilistic nature in time-varying delays. By composing a suitable Lyapunov-Krasovskii functional (LKF) containing triple summation terms with the aid of Kronecker product properties and utilizing free weighting matrix approach, sufficient criteria are established in terms of linear matrix inequalities (LMIs) that assures finite-time synchronization and meets the dissipativity performance to the addressed CDNs. The usefulness of the presented design scheme is finally verified by numerical examples.
\end{abstract}

Keywords: Finite-time synchronization, Complex dynamical networks, Dissipativity, Semi-Markovian jumping, Reliable control.

\footnotetext{
*Corresponding author: ykma@kongju.ac.kr (Yong-Ki Ma)
} 


\section{Introduction}

Complex network is a large-scale network composed of a large number of nodes joined by edges, and it can mirror several important structure characteristics and dynamic performances of real networks [1,2]. In recent years, the study on CDNs serve as one of the most active research field in control theory that have found successful applications in real world disciplines such as supply chain and manufacturing networks, electricity power grids, transportation systems, water distribution systems, gas transmission, world wide web, social interaction, biological networks, co-authorship and citation of networks of scientists. Due to these utilizations, the researchers have been devoting their interest enormously to investigate the control problems and dynamical behaviors of CDNs [1]- [4].

On the other hand, in practice, synchronization is interesting and significant dynamic phenomena of CDNs compared to other dynamical performance such as auto-waves, spatio-temporal chaos, self-organization and spiral waves etc. Inspired by these advantages, the research communities have investigated synchronization of a CDNs presented in [5]- [7]. Li et al. [6] studied the synchronization problem for a class of discrete-time CDNs with partial mixed impulsive effects. But in practice, the networks might be expected to achieve synchronization in finite-time interval rather than asymptotically. To achieve faster convergence rate in time delay complex networks, an effective method called finite-time synchronization or finite-time boundedness can be used. In addition, finite-time control design have been proposed for the synchronization of different CDNs as authorized by various literatures [8]- [10]. The finite-horizon bounded $H_{\infty}$ synchronization and state estimation problem for a class of discrete-time complex networks with missing measurements is reported in [8].

Moreover, few actuators may be faulty and uniform of inferior quality, the CDNs structure may be changed as well as the closed loop system becomes unstable. To improve system reliability and security, it is most important to design a reliable controller so that the stability and the performance of the Takagi-Sugeno fuzzy closed-loop system can operate fine, even in the existence of some actuator faults in [11]. Consequently, the study on fault tolerant control or reliable control for dynamical systems has received many results, see for example [12]- [14]. Naturally, the inclusion of time delays are inevitable phenomenon in all kinds of dynamical systems and it arises due to the finite propagation speed between nodes, traffic congestions and memory effects, it has the capability to alter notoriously the dynamic behavior of the system such as stability. Therefore, much work has been done for synchronization of discrete-time networks with time-delay [15]- [17]. The existence of time delay in a stochastic fashion in some practical systems, it consequences the researchers have focused the synchronization analysis of CDNs with probabilistic time-varying delay [18]- [20]. The problem of cluster synchronization in finite time complex networks with probabilistic coupling delays has been addressed in [18]. 
In most of the practical networks, the abrupt variations often expose in system dynamics due to random failures, changes in the subsystem interconnections and information latching. Therefore, in order to tackle this issues, it is essential to describe CDNs with Markovian jumping parameters, where each state denotes in a discrete-time system with finite discrete jumping mode governed by the Markov process and few interesting results regarding Markov jump systems have been addressed in [21]- [23]. It is pointed that the above literatures have dealt with transition rates of Markov process are constants due to that the sojourn time between two jumps of the Markov chain is governed by exponential distribution. Also, it should be mentioned that the Markov process might consist of time-varying transition rates when modeling practical systems, such kind of process is known as semi-Markov process. Apparently, semi-Markov jump CDNs are comparatively more general than the Markov jump CDNs. Shen et al. [24] obtained the finite-time $H_{\infty}$ synchronization criterion for complex networks with time-varying delays and semi-Markov jump topology. The existence of sufficient conditions in [25] and convex optimization technique which ensure the $L_{2}-L_{\infty}$ synchronization for singularly perturbed complex networks subject to semi-Markov jump topology.

Furthermore, the dissipativity theory was introduced by Willems [26], it serves as a powerful tool in control applications such as robotics, active vibration damping, electromechanical system and circuit theory. Generally speaking, dissipativity which postulates the quantity of energy supplied from the external source is not less than the energy lost inside the dynamic system [27]. The main idea of dissipative theory is the dissipative quality of a system can keep the system internally stable. Also, the dissipativity system theory is a more general criterion when compared with passivity and $H_{\infty}$ performance. In [28], a set of sufficient conditions is established by using Lyapunov functional methodlogy and completing square technique for dissipativity control of discrete nonlinear Markovian jump systems with discrete and distributed time-delays. Wang et al. [29], addresses the problem of generalized dissipativity-based synchronization for complex networks with semi-Markov jump topology. In spite, up to now, the finite-time dissipative synchronization has not yet been reported for a class of discretetime semi-Markovian jump CDNs.

Motivated by the above discussions, this paper analyzes finite-time synchronization problem for discrete-time semi-Markovian jumping CDNs with probabilistic time-varying delay components based on dissipative performance. The reliable control strategy and probability distribution of the time-varying delays are proposed. By constructing a new LKF and Kronecker product technique, delay dependent sufficient conditions under which the CDNs are synchronized to the given finite-time interval. The derived conditions depend not only on the size of the delay but also on the probability of the delay taking values in some intervals. Based on this derived condition, a design algorithm of the proposed state feedback controller which ensure the finite-time synchronization of the CDNs with actuator faults. Finally numerical examples are provided to illustrate the effectiveness of our 
theoretical results.

Notation: Throughout this paper, superscripts " $T$ " and " $(-1)$ " stand for matrix transposition and matrix inverse, respectively. $\mathbb{R}^{n}$ denotes the $n$-dimensional Euclidean space and $\mathbb{R}^{n \times n}$ denotes the set of all $n \times n$ real matrices. $P>0$ and $P<0$ represent positive definite and negative definite, respectively. $L_{2}[0, \infty)$ stands for the space of $n$-dimensional square integrable function over $[0, \infty)$. $I$ and 0 represent identity matrix and zero matrix with compatible dimensions. The asterisk "* represents a term that is induced by symmetry. $\operatorname{diag}\{\ldots\}$ stands for a block-diagonal matrix. The Kronecker product of matrices $S \in \mathbb{R}^{m \times n}$ and $T \in \mathbb{R}^{p \times q}$ is a matrix in $\mathbb{R}^{m p \times n q}$ and denoted as $S \otimes T$.

\section{Network model and Preliminaries}

Consider the following discrete-time semi-Markovian jumping CDNs consisting of $\mathrm{N}$ nonlinearly coupled nodes, with each node being of $\mathrm{n}$ dimensional and have identical dynamic performance. It can be designated as

$$
\begin{aligned}
x_{l}(k+1)= & A(\sigma(k)) x_{l}(k)+B(\sigma(k)) f\left(k, x_{l}(k)\right)+C(\sigma(k)) f\left(k, x_{l}(k-d(k))\right)+\sum_{m=1}^{N} d_{l m}(\sigma(k)) \Gamma_{1} \\
& x_{m}(k)+\sum_{m=1}^{N} g_{l m}(\sigma(k)) \Gamma_{2} x_{m}(k-\tau(k))+R(\sigma(k)) V_{l}^{f}(k)+w(k) \\
z_{l}(k)= & J(\sigma(k)) x_{l}(k), \quad l=1,2, \ldots, N
\end{aligned}
$$

where $x_{l}(k) \in \mathbb{R}^{n}$ denotes the state vector of the $l^{\text {th }}$ node; $A(\sigma(k)), B(\sigma(k)), C(\sigma(k)), R(\sigma(k))$ and $J(\sigma(k))$ are constant matrices with suitable dimensions at instant $\mathrm{k} ; f\left(k, x_{l}(k)\right)$ and $f\left(k, x_{l}(k-d(k))\right.$ represent the nonlinear vector-valued functions without and with time delays respectively. $d_{l m}(\sigma(k))$, $g_{l m}(\sigma(k))$ are the elements of the outer coupling configuration matrices $D(\sigma(k))$ and $G(\sigma(k))$ which describes the coupling structure of the CDNs. The positive diagonal matrices $\Gamma_{1}$ and $\Gamma_{2}$ are denotes inner coupling matrices with suitable dimension; $V_{l}^{f}(k)$ is the control input of the $l^{\text {th }}$ node. If there is a link from node $l$ to node $m$, then $d_{l m}(\sigma(k))>0, g_{l m}(\sigma(k))>0$; otherwise $d_{l m}(\sigma(k))=g_{l m}(\sigma(k))=0$.

Further, the diagonal elements of the outer coupling matrices are given as follows: $g_{l l}(\sigma(k))=$ - $\sum_{m=1 ; l \neq m}^{N} g_{l m}(\sigma(k)) ; w(k)$ denotes the disturbance input which satisfies $w^{T}(k) w(k) \leq \delta ; z(k)$ is the objective output to be estimated.

Let $\sigma(k), k \leq 0$ is a discrete-time semi-Markov process and take values from a finite set $\psi=\{1,2, \cdots, N\}$ with transition probabilities $P\{\sigma(k+1)=q \mid \sigma(k)=p\}=\Pi_{p q}$, where $0 \leq \Pi_{p q} \leq 1$ for all $p, q \in \psi$ 
and $\sum_{q \in \psi} \Pi_{p q}=1$. For notational simplicity, we take $\sigma(k)=p$.

Assumption 1: Considering the information of probability distribution of the time delays $d(k)$ and $\tau(k)$, we define

$$
\begin{array}{ll}
P\left\{d(k) \in\left[d_{m}, d_{0}\right]\right\}=\beta_{0}, & P\left\{d(k) \in\left(d_{0}, d_{M}\right]\right\}=1-\beta_{0}, \\
P\left\{\tau(k) \in\left[\tau_{m}, \tau_{0}\right]\right\}=\alpha_{0}, & P\left\{\tau(k) \in\left(\tau_{0}, \tau_{M}\right]\right\}=1-\alpha_{0},
\end{array}
$$

where $0 \leq \alpha_{0}, \beta_{0} \leq 1$ are constants and $d_{0}, \tau_{0}$ are integers satisfying $d_{m} \leq d_{0}<d_{M}, \tau_{m} \leq \tau_{0}<\tau_{M}$. Therefore the stochastic variable $\alpha(k), \beta(k)$ can be defined as

$$
\alpha(k)=\left\{\begin{array}{ll}
1, & \tau(k) \in\left[\tau_{m}, \tau_{0}\right] \\
0, & \tau(k) \in\left(\tau_{0}, \tau_{M}\right]
\end{array} \quad \beta(k)= \begin{cases}1, & d(k) \in\left[d_{m}, d_{0}\right] \\
0, & d(k) \in\left(d_{0}, d_{M}\right]\end{cases}\right.
$$

Assumption 2: $\beta(k)$ and $\alpha(k)$ are the Bernoulli distributed sequences with

$$
\begin{aligned}
& P\{\beta(k)=1\}=P\left\{d(k) \in\left[d_{m}, d_{0}\right]\right\}=E\{\beta(k)\}=\beta_{0}, \\
& P\{\beta(k)=0\}=P\left\{d(k) \in\left(d_{0}, d_{M}\right]\right\}=1-E\{\beta(k)\}=1-\beta_{0}, \\
& P\{\alpha(k)=1\}=P\left\{\tau(k) \in\left[\tau_{m}, \tau_{0}\right]\right\}=E\{\alpha(k)\}=\alpha_{0}, \\
& P\{\alpha(k)=0\}=P\left\{\tau(k) \in\left(\tau_{m}, \tau_{0}\right]\right\}=E\{\alpha(k)\}=\alpha_{0} .
\end{aligned}
$$

where $\mathbb{E}\{\beta(k)\}$ and $\mathbb{E}\{\alpha(k)\}$ are the expectations of $\beta(k)$ and $\alpha(k)$, respectively.

From the Assumption 1, it is easy to say that

$$
\begin{aligned}
& \mathbb{E}\left\{\beta(k)-\beta_{0}\right\}=0, \quad \mathbb{E}\left\{\left(\beta(k)-\beta_{0}\right)^{2}\right\}=\beta_{0}\left(1-\beta_{0}\right), \\
& \mathbb{E}\left\{\alpha(k)-\alpha_{0}\right\}=0, \quad \mathbb{E}\left\{\left(\alpha(k)-\alpha_{0}\right)^{2}\right\}=\alpha_{0}\left(1-\alpha_{0}\right) .
\end{aligned}
$$

Next, the discrete delays $d_{1}(k), d_{2}(k), \tau_{1}(k)$ and $\tau_{2}(k)$ are introduced in the following manner:

$$
d(k)=\left\{\begin{array}{ll}
d_{1}(k), & d(k) \in\left[d_{m}, d_{0}\right] \\
d_{2}(k), & d(k) \in\left(d_{0}, d_{M}\right]
\end{array} \quad \tau(k)= \begin{cases}\tau_{1}(k), & \tau(k) \in\left[\tau_{m}, \tau_{0}\right] \\
\tau_{2}(k), & \tau(k) \in\left(\tau_{0}, \tau_{M}\right] .\end{cases}\right.
$$

By using Assumptions 1 and 2, CDNs (1) can be rewritten as

$$
\begin{aligned}
x_{l}(k+1)= & A_{p} x_{l}(k)+B_{p} f\left(k, x_{l}(k)\right)+\beta_{0} C_{p} f\left(k, x_{l}\left(k-d_{1}(k)\right)\right)+\left(1-\beta_{0}\right) C_{p} f\left(k,\left(x_{l}\left(k-d_{2}(k)\right)\right)\right) \\
& +\left(\beta(k)-\beta_{0}\right)\left[C_{p} f\left(k, x_{l}\left(k-d_{1}(k)\right)\right)-C_{p} f\left(k, x_{l}\left(k-d_{2}(k)\right)\right)\right]+\sum_{m=1}^{N} d_{l m p} \Gamma_{1} x_{m}(k) \\
& +\alpha_{0} \sum_{m=1}^{N} g_{l m p} \Gamma_{2} x_{m}\left(k-\tau_{1}(k)\right)+\left(1-\alpha_{0}\right) \sum_{m=1}^{N} g_{l m p} \Gamma_{2} x_{m}\left(k-\tau_{2}(k)\right)+\left(\alpha(k)-\alpha_{0}\right) \\
& \times\left[\sum_{m=1}^{N} g_{l m p} \Gamma_{2} x_{m}\left(k-\tau_{1}(k)\right)-\sum_{m=1}^{N} g_{l m p} \Gamma_{2} x_{m}\left(k-\tau_{2}(k)\right)\right]+R_{p} V_{l}^{f}(k)+w(k),
\end{aligned}
$$


$z_{l}(k)=J_{p} x_{l}(k)$.

Assumption 3: For the nonlinear function $f\left(k, x(k), x\left(k-d_{1}(k)\right), x\left(k-d_{2}(k)\right)\right.$, there exists the known real constant matrix $\mathscr{K}_{1}, \mathscr{K}_{2}$ and $\mathscr{K}_{3}$ such that $\|f(k, x(k))\| \leq \mathscr{K}_{1}\|x(k)\|, \| f(k, x(k-$ $\left.\left.d_{1}(k)\right)\right)\left\|\leq \mathscr{K}_{2}\right\| x\left(k-d_{1}(k)\right) \|$ and $\left\|f\left(k, x\left(k-d_{2}(k)\right)\right)\right\| \leq \mathscr{K}_{3}\left\|x\left(k-d_{2}(k)\right)\right\|$ for any $x(k) \in \mathbb{R}^{n}$.

Let $e_{l}(k)=x_{l}(k)-s(k)$ be the synchronization error, where $s(k)$ be a solution of an isolated node which is described as

$$
\begin{aligned}
s(k+1)= & A_{p} s(k)+B_{p} f(k, s(k))+\beta_{0} C_{p} f\left(k, s\left(k-d_{1}(k)\right)\right)+\left(1-\beta_{0}\right) C_{p} f\left(k, s\left(k-d_{2}(k)\right)\right) \\
& +\left(\beta(k)-\beta_{0}\right)\left[C_{p} f\left(k, s\left(k-d_{1}(k)\right)\right)-C_{p} f\left(k, s\left(k-d_{2}(k)\right)\right)\right] .
\end{aligned}
$$

Then the corresponding error dynamics of the CDNs (1) can be obtained as follows:

$$
\begin{aligned}
e_{l}(k+1)= & A_{p} e_{l}(k)+B_{p} F\left(k, e_{l}(k)\right)+\beta_{0} C_{p} F\left(k, e_{l}\left(k-d_{1}(k)\right)\right)+\left(1-\beta_{0}\right) C_{p} F\left(k, e_{l}\left(k-d_{2}(k)\right)\right) \\
& +\left(\beta(k)-\beta_{0}\right)\left[C_{p} F\left(k, e_{l}\left(k-d_{1}(k)\right)\right)-C_{p} F\left(k, e_{l}\left(k-d_{2}(k)\right)\right)\right]+\sum_{m=1}^{N} d_{l m p} \Gamma_{1} e_{m}(k) \\
& +\alpha_{0} \sum_{m=1}^{N} g_{l m p} \Gamma_{2} e_{m}\left(k-\tau_{1}(k)\right)+\left(1-\alpha_{0}\right) \sum_{m=1}^{N} g_{l m p} \Gamma_{2} e_{m}\left(k-\tau_{2}(k)\right)+\left(\alpha(k)-\alpha_{0}\right) \\
& \times\left[\sum_{m=1}^{N} g_{l m p} \Gamma_{2} e_{m}\left(k-\tau_{1}(k)\right)-\sum_{m=1}^{N} g_{l m p} \Gamma_{2} e_{m}\left(k-\tau_{2}(k)\right)\right]+R_{p} V_{l}^{f}(k)+w(k), \\
z_{l}(k)= & J_{p} e_{l}(k),
\end{aligned}
$$

where $F\left(k, e_{l}(k)\right)=f\left(k, x_{l}(k)\right)-f(k, s(k))$.

Now consider the actuator fault model, for the control input $V_{l}^{f}(k)$. We designate $V_{l}^{f}(k)$ to describe the signal sent from the actuator and satisfies

$$
V_{l}^{f}(k)=\mathbb{G} V_{l}(k),
$$

where $\mathbb{G}$ is the actuator fault matrix defined as $\mathbb{G}=\operatorname{diag}\left\{g_{1}, g_{2}, \cdots, g_{m}\right\}, 0 \leq \underline{g}_{i} \leq g_{i} \leq \bar{g}_{i} \leq 1$, where $\underline{g}_{i}$ and $\bar{g}_{i}, i=1,2, \cdots, m$ are given constants. $g_{i}=0$ means that $i$ th actuator fails, $g_{i}=1$ means that $i^{\text {th }}$ actuator is normal, when $0<g_{i}<1$ denotes the $i^{\text {th }}$ actuator meets partial failures. In this paper, we designing a state feedback controller of the form:

$$
V_{l}(k)=K_{p} e_{l}(k)
$$

where $K_{p}$ is the state feedback controller gain matrix to be determined. Then, by substituting (5) in (4) and using Kronecker product properties, the resulting closed loop system can be written as $e(k+1)=\bar{A} e(k)+\bar{B} F(k, e(k))+\beta_{0} \bar{C} F\left(k, e\left(k-d_{1}(k)\right)\right)+\left(1-\beta_{0}\right) \bar{C} F\left(k, e\left(k-d_{2}(k)\right)\right)$ 


$$
\begin{aligned}
& +\left(\beta(k)-\beta_{0}\right)\left[\bar{C} F\left(k, e\left(k-d_{1}(k)\right)\right)-\bar{C} F\left(k, e\left(k-d_{2}(k)\right)\right)\right]+\bar{D} e(k)+\alpha_{0} \bar{G} e\left(k-\tau_{1}(k)\right) \\
& +\left(1-\alpha_{0}\right) \bar{G}\left(e\left(k-\tau_{2}(k)\right)\right)+\left(\alpha(k)-\alpha_{0}\right)\left[\bar{G}\left(e\left(k-\tau_{1}(k)\right)\right)-\bar{G}\left(e\left(k-\tau_{2}(k)\right)\right)\right] \\
& +\bar{R} \mathbb{G} K_{p} e(k)+w(k) \\
z(k)= & \bar{J} e(k) .
\end{aligned}
$$

where $\bar{A}=\left(I \otimes A_{p}\right), \bar{B}=\left(I \otimes B_{p}\right), \bar{C}=\left(I \otimes C_{p}\right), \bar{D}=\left(D_{p} \otimes \Gamma_{1}\right), \bar{G}=\left(G_{p} \otimes \Gamma_{2}\right), \bar{R}=\left(I \otimes R_{p}\right)$, $\bar{J}=\left(I \otimes J_{p}\right), e(t)=\left[e_{1}^{T}(t), e_{2}^{T}(t), \cdots, e_{N}^{T}(t)\right]^{T}, F(k, e(k))=\left[F^{T}\left(k, e_{1}(k)\right), F^{T}\left(k, e_{2}(k)\right), \cdots\right.$, $\left.F^{T}\left(k, e_{N}(k)\right)\right]^{T}, F\left(k, e\left(k-\tau_{1}(k)\right)\right)=\left[F^{T}\left(k, e_{1}\left(k-\tau_{1}(k)\right)\right), F^{T}\left(k, e_{2}\left(k-\tau_{1}(k)\right)\right), \cdots, F^{T}\left(k, e_{N}(k-\right.\right.$ $\left.\left.\left.\tau_{1}(k)\right)\right)\right]^{T}, F\left(k, e\left(k-\tau_{2}(k)\right)\right)=\left[F^{T}\left(k, e_{1}\left(k-\tau_{2}(k)\right)\right), F^{T}\left(k, e_{2}\left(k-\tau_{2}(k)\right)\right), \cdots, F^{T}\left(k, e_{N}(k-\right.\right.$ $\left.\left.\left.\tau_{2}(k)\right)\right)\right]^{T}$.

Further, we introduce the following lemmas and definitions, which will be important for the derivations of the main results.

Lemma 1(Schur complement) [30]. Given constant matrices $E_{11}, E_{12}$ and $E_{22}$ with appropriate dimen sions where $E_{11}^{T}=E_{11}$ and $E_{22}^{T}=E_{22}$ then $E_{11}+E_{12}^{T} E_{22}^{-1} E_{12}<0$ iff $\left[\begin{array}{cc}E_{11} & E_{12}^{T} \\ * & -E_{22}\end{array}\right]<0$.

Definition 1 [30]. The error dynamics [6], subject to an exogeneous disturbance $w(k)$ satisfy $\sum_{k=0}^{N} w^{T}(k)$ $w(k)<\delta$ is said to be stochastically finite-time bounded(SFTB) with respect to $\left(c_{1}, c_{2}, S_{p}, \mathbb{N}, \delta\right)$, where $S_{p}(p \in \psi)$ is a positive definite matrix, $0<c_{1}<c_{2}, \delta>0$ if $E\left\{e^{T}\left(k_{1}\right)\left(I \otimes S_{p}\right) e\left(k_{1}\right)\right\} \leq$ $c_{1} \Longrightarrow E\left\{e^{T}\left(k_{2}\right)\left(I \otimes S_{p}\right) e\left(k_{2}\right)\right\} \leq c_{2}, \forall k_{1} \in\{-d,-d+1, \cdots, 0\}, \forall k_{2} \in\{1,2, \cdots, \mathbb{N}\}$, where $d=\max \left\{d_{M}, \tau_{M}\right\}$.

Definition 2 [31]. The error dynamics (6) is said to be stochastically finite-time stable(SFTS) with respect to $\left(c_{1}, c_{2}, S_{p}, \mathbb{N}\right)$,

where $S_{p}$ is a positive definite matrix, $0<c_{1}<c_{2}$ and $w(k)=0$ if $E\left\{e^{T}\left(k_{1}\right)\left(I \otimes S_{p}\right) e\left(k_{1}\right)\right\} \leq$ $c_{1} \Longrightarrow E\left\{e^{T}\left(k_{2}\right)\left(I \otimes S_{p}\right) e\left(k_{2}\right)\right\} \leq c_{2}, \forall k_{1} \in\left\{-d_{M},-d_{M}+1, \cdots, 0\right\}, \forall k_{2} \in\{1,2, \cdots, \mathbb{N}\}$.

Definition $3[32]$. The error dynamics $[6]$ is $(\mathbb{L}, \mathbb{M}, \mathbb{R})-\gamma$ dissipative with respect to $\left(c_{1}, c_{2}, S_{p}, \mathbb{N}, \delta\right)$, where $0<c_{1}<c_{2}, \gamma>0$ and $S_{p}$ is a positive definite matrix, if the system is stochastically finitetime bounded(SFTB) with respect to $\left(c_{1}, c_{2}, S_{p}, \mathbb{N}, \delta\right)$ and under the zero initial condition, the output $z(k)$ satisfies

$$
\sum_{k=0}^{\mathbb{N}}\left[e^{t}(k) \mathbb{L} e(k)+2 e^{T}(k) \mathbb{M} w(k)+w^{T}(k) \mathbb{R} w(k)\right] \geq \gamma \sum_{k=0}^{\mathbb{N}} w^{T}(k) w(k),
$$

for any non-zero satisfies $\sum_{k=0}^{N} w^{T}(k) w(k)<\delta$, here $\mathbb{L}, \mathbb{M}$ and $\mathbb{R}$ are real-valued matrices of appropriate dimensions with $\mathbb{L}$ and $\mathbb{Q}$ are symmetric. Without loss of generality, it is assume that $\mathbb{L} \leq 0$, then we have $-\mathbb{L}=\left(\overline{\mathbb{L}}^{1 / 2}\right)^{2}$.

Remark 1 In the view points of the above definitions, it should be noted that the SFTS of the errror 
system (6) can guarantee the SFTB. That is, in the absence of exogenous disturbance inputs $w(k)=$ 0 , the concept of SFTB is reduced to SFTS. Thus, SFTB implies SFTS but the converse is not true. Consequently, the system (1) is finite time synchronized under the feedback control law (5).

\section{MAIN RESULTS}

In this section, to design a state feedback controller which ensures the stochastic synchronization in a finite-time interval of the CDNs (1). First we find the stochastic finite-time boundedness for the closed loop system (6). Next, we find the stochastic finite-time boundedness with dissipativity performance index.

\section{A. Stochastic finite-time boundedness}

Theorem 1. Under Assumptions 1 and 2, given scalars $\mu \geq 1, \kappa_{i}>0(i=1,2)$ and known actuator fault matrix $\mathbb{G}$, the system $(6)$ is stochastic finite-time bounded with respect to $\left(c_{1}, c_{2}, S_{p}, \mathbb{N}, \delta\right)$, if there exists symmetric matrices $P_{p}>0(p \in \psi), Q_{b}>0(b=1,2, \cdots, 6), T_{c}>0$ and $U_{c}>0(c=1,2), V_{l}>0(l=1,2,3,4)$ such that the following inequalities holds:

$$
\begin{gathered}
\Psi=\left[\begin{array}{ccc}
\Gamma_{20 \times 20} & \Xi_{1} & \Xi_{2} \\
* & -I & 0 \\
* & * & \Xi_{3}
\end{array}\right]<0 \\
\zeta_{1} c_{1}+\lambda_{w} \delta<\lambda_{1} c_{2} \mu^{-k}
\end{gathered}
$$

where $\Gamma_{1,1}=-\mu\left(I \otimes P_{p}\right)+\left(I \otimes Q_{1}\right)+\left(I \otimes Q_{2}\right)+\left(I \otimes Q_{3}\right)+\left(I \otimes Q_{4}\right)+\left(I \otimes Q_{5}\right)++\left(I \otimes Q_{6}\right)+\left(d_{0}-\right.$ $\left.d_{m}+1\right)\left(I \otimes T_{1}\right)+\left(\tau_{0}-\tau_{m}+1\right)\left(I \otimes T_{2}\right)+\left(d_{M}-d_{0}+1\right)\left(I \otimes U_{1}\right)+\left(\tau_{M}-\tau_{0}+1\right)\left(I \otimes U_{2}\right)+d_{m}(I \otimes$ $\left.V_{1}\right)+d_{M}\left(I \otimes V_{3}\right)+\tau_{m}\left(I \otimes V_{3}\right)+\tau_{M}\left(I \otimes V_{4}\right)+\kappa_{1}\left(I \otimes P_{p}\right)\left(\bar{A}+\bar{R} \mathbb{G}_{K_{p}}+\bar{D}-1\right)+\left(\bar{A}+\bar{R} \mathbb{G} K_{p}+\bar{D}-\right.$ $1)^{T}\left(I \otimes P_{p}\right)^{T} \kappa_{1}, \quad \Gamma_{1,10}=\alpha_{0} \kappa_{1}\left(I \otimes P_{p}\right) \bar{G}, \Gamma_{1,11}=\kappa_{1}\left(1-\alpha_{0}\right)\left(I \otimes P_{p}\right) \bar{G}, \Gamma_{1,12}=\kappa_{1}\left(I \otimes P_{p}\right) \bar{B}$, $\Gamma_{1,13}=\kappa_{1} \beta_{0}\left(I \otimes P_{p}\right) \bar{C}, \Gamma_{1,14}=\kappa_{1}\left(1-\beta_{0}\right)\left(I \otimes P_{p}\right) \bar{C}, \Gamma_{1,19}=-\kappa_{1}\left(I \otimes P_{p}\right)+\left(\bar{A}+\bar{R} \mathbb{G}_{p}+\bar{D}-\right.$ $1)^{T}\left(I \otimes P_{p}\right)^{T} \kappa_{2}, \Gamma_{1,20}=\kappa_{1}\left(I \otimes P_{p}\right), \Gamma_{2,2}=-\left(I \otimes Q_{1}\right), \Gamma_{3,3}=-\left(I \otimes Q_{2}\right), \Gamma_{4,4}=-\left(I \otimes Q_{3}\right)$, $\Gamma_{5,5}=-\left(I \otimes T_{1}\right), \Gamma_{6,6}=-\left(I \otimes U_{1}\right), \Gamma_{7,7}=-\left(I \otimes Q_{4}\right), \Gamma_{8,8}=-\left(I \otimes Q_{5}\right), \Gamma_{9,9}=-\left(I \otimes Q_{6}\right)$, $\Gamma_{10,10}=-\left(I \otimes T_{2}\right), \Gamma_{10,19}=\alpha_{0} \kappa_{2} \bar{G}^{T}\left(I \otimes P_{p}\right), \Gamma_{11,11}=-\left(I \otimes U_{2}\right), \Gamma_{11,19}=\left(1-\alpha_{0}\right) \kappa_{2} \bar{G}^{T}\left(I \otimes P_{p}\right)$, $\Gamma_{12,12}=-I, \Gamma_{12,19}=\kappa_{2} \bar{B}^{T}\left(I \otimes P_{p}\right)^{T}, \Gamma_{13,13}=-I, \Gamma_{13,19}=\kappa_{2} \beta_{0} \bar{C}^{T}\left(I \otimes P_{p}\right), \Gamma_{14,14}=-I$, $\Gamma_{14,19}=\kappa_{2}\left(1-\beta_{0}\right) \bar{C}^{T}\left(I \otimes P_{p}\right), \Gamma_{15,15}=-1 / d_{m}\left(I \otimes V_{1}\right), \Gamma_{16,16}=-1 / d_{M}\left(I \otimes V_{2}\right), \Gamma_{17,17}=$ $-1 / \tau_{m}\left(I \otimes V_{3}\right), \Gamma_{18,18}=-1 / \tau_{M}\left(I \otimes V_{4}\right), \Gamma_{19,19}=-\kappa_{2}\left(I \otimes P_{p}\right)-\kappa_{2}\left(I \otimes P_{p}\right)^{T}, \Gamma_{20,20}=-\bar{W}$, $\Xi_{1}=[(I \otimes W), 0,0,0,(I \otimes E),(I \otimes H), \underbrace{0 \cdots 0}_{14}], \quad \Xi_{2}=\left[\Xi_{21} \Xi_{22} \cdots \Xi_{2 N}\right], \quad \Xi_{21}=\left[\sqrt{\Pi_{p 1}}(I \otimes\right.$ 
$\left.P_{1}\right) \underbrace{0 \cdots 0}_{17}, \sqrt{\Pi}_{p 1}\left(I \otimes P_{1}\right), 0]^{T}, \Xi_{22}=[\sqrt{\Pi}_{p 2}\left(I \otimes P_{2}\right) \underbrace{0 \cdots 0}_{17}, \sqrt{\Pi}_{p 2}\left(I \otimes P_{2}\right), 0]^{T}, \cdots, \Xi_{2 N}=$ $[\sqrt{\Pi}_{p N}\left(I \otimes P_{N}\right) \underbrace{0 \cdots 0}_{17}, \sqrt{\Pi}_{p N}\left(I \otimes P_{N}\right), 0]^{T}, \Xi_{3}=-\operatorname{diag}\left\{\left(I \otimes P_{1}\right),\left(I \otimes P_{2}\right), \cdots,\left(I \otimes P_{N}\right)\right\}$, $\lambda_{1}=\min \left\{\lambda_{\min }\left(I \otimes \mathscr{P}_{p}\right), p \in \psi\right\}, \lambda_{2}=\max \left\{\lambda_{\max }\left(I \otimes \mathscr{P}_{p}\right), p \in \psi\right\}, \lambda_{3}=\max \left\{\lambda_{\max }(I \otimes\right.$ $\left.\left.\mathscr{Q}_{1 p}\right), p \in \psi\right\}, \quad \lambda_{4}=\max \left\{\lambda_{\max }\left(I \otimes \mathscr{Q}_{2 p}\right), p \in \psi\right\}, \quad \lambda_{5}=\max \left\{\lambda_{\max }\left(I \otimes \mathscr{Q}_{3 p}\right), p \in \psi\right\}$, $\lambda_{6}=\max \left\{\lambda_{\max }\left(I \otimes \mathscr{Q}_{4 p}\right), p \in \psi\right\}, \lambda_{7}=\max \left\{\lambda_{\max }\left(I \otimes \mathscr{Q}_{5 p}\right), p \in \psi\right\}, \lambda_{8}=\max \left\{\lambda_{\max }(I \otimes\right.$ $\left.\left.\mathscr{Q}_{6 p}\right), p \in \psi\right\}, \quad \lambda_{9}=\max \left\{\lambda_{\max }\left(I \otimes \mathscr{T}_{1 p}\right), p \in \psi\right\}, \quad \lambda_{10}=\max \left\{\lambda_{\max }\left(I \otimes \mathscr{T}_{2 p}\right), p \in \psi\right\}$, $\lambda_{11}=\max \left\{\lambda_{\max }\left(I \otimes \mathscr{U}_{1 p}\right), p \in \psi\right\}, \lambda_{12}=\max \left\{\lambda_{\max }\left(I \otimes \mathscr{U}_{2 p}\right), p \in \psi\right\}, \lambda_{13}=\max \left\{\lambda_{\max }(I \otimes\right.$ $\left.\left.\mathscr{V}_{1 p}\right), p \in \psi\right\}, \quad \lambda_{14}=\max \left\{\lambda_{\max }\left(I \otimes \mathscr{V}_{2 p}\right), p \in \psi\right\}, \lambda_{15}=\max \left\{\lambda_{\max }\left(I \otimes \mathscr{V}_{3 p}\right), p \in \psi\right\}, \quad \lambda_{16}=$ $\max \left\{\lambda_{\max }\left(I \otimes \mathscr{V}_{4 p}\right), p \in \psi\right\}, \zeta_{1}=\lambda_{2}+\lambda_{3} d_{m}+\lambda_{4} d_{0}+\lambda_{5} d_{M}+\lambda_{6} \tau_{m}+\lambda_{7} \tau_{0}+\tau_{8} \tau_{M}+\lambda_{9} d_{0}+$ $\lambda_{9} \frac{\left(d_{0}-d_{m}\right)\left(d_{m}+d_{0}-1\right)}{2}+\lambda_{10} \tau_{0}+\lambda_{10} \frac{\left(\tau_{0}-\tau_{m}\right)\left(\tau_{m}+\tau_{0}-1\right)}{2}+\lambda_{11} d_{M}+\lambda_{11} \frac{\left(d_{0}+d_{m}\right)\left(d_{m}-d_{0}+1\right)}{2}+\lambda_{12} \tau_{M}+$ $\lambda_{12} \frac{\left(\tau_{0}+\tau_{m}\right)\left(\tau_{m}-\tau_{0}+1\right)}{2}+\lambda_{13} \frac{d_{m}\left(d_{m}-1\right)}{2}+\lambda_{14} \frac{d_{M}\left(d_{M}-1\right)}{2}+\lambda_{15} \frac{\tau_{m}\left(\tau_{m}-1\right)}{2}+\lambda_{16} \frac{\tau_{M}\left(\tau_{M}-1\right)}{2}$.

Proof: Construct the following LKF for the error system (6) as

$$
\mathcal{V}(k)=\sum_{b=1}^{5} \mathcal{V}_{b}(k)
$$

where

$$
\begin{aligned}
& \mathcal{V}_{1}(k)=e^{T}(k)\left(I \otimes P_{p}\right) e(k), \\
& \mathcal{V}_{2}(k)=\sum_{j=k-d_{m}}^{k-1} e^{T}(j)\left(I \otimes Q_{1}\right) e(j)+\sum_{j=k-d_{0}}^{k-1} e^{T}(j)\left(I \otimes Q_{2}\right) e(j)+\sum_{j=k-d_{M}}^{k-1} e^{T}(j)\left(I \otimes Q_{3}\right)(k) e(j) \\
& +\sum_{j=k-\tau_{m}}^{k-1} e^{T}(j)\left(I \otimes Q_{4}\right) e(j)+\sum_{j=k-\tau_{0}}^{k-1} e^{T}(j)\left(I \otimes Q_{5}\right) e(s)+\sum_{j=k-\tau_{M}}^{k-1} e^{T}(j)\left(I \otimes Q_{6}\right) e(j), \\
& \mathcal{V}_{3}(k)=\sum_{j=k-d_{1}(k)}^{k-1} e^{T}(j)\left(I \otimes T_{1}\right) e(j)+\sum_{j=d_{m}}^{d_{0}-1} \sum_{l=k-j}^{k-1} e^{T}(l)\left(I \otimes T_{1}\right) e(l)+\sum_{j=k-\tau_{1}(k)}^{k-1} e^{T}(j)\left(I \otimes T_{2}\right) e(j) \\
& +\sum_{j=\tau_{m}}^{\tau_{0}-1} \sum_{l=k-j}^{k-1} e^{T}(l)\left(I \otimes T_{2}\right) e(l) \\
& \mathcal{V}_{4}(k)=\sum_{j=k-d_{2}(k)}^{k-1} e^{T}(j)\left(I \otimes U_{1}\right) e(j)+\sum_{j=d_{0}+1}^{d_{m}-1} \sum_{l=k-j}^{k-1} e^{T}(l)\left(I \otimes U_{1}\right) e(l)+\sum_{s=k-\tau_{2}(k)}^{k-1} e^{T}(j)\left(I \otimes U_{2}\right) e(j) \\
& +\sum_{j=\tau_{0}+1}^{\tau_{m}-1} \sum_{l=k-j}^{k-1} e^{T}(l)\left(I \otimes U_{2}\right) e(l), \\
& \mathcal{V}_{5}(k)=\sum_{j=k-d_{m}}^{k-1} \sum_{l=j}^{k-1} e^{T}(l)\left(I \otimes V_{1}\right) e(l)+\sum_{j=k-d_{M}}^{k-1} \sum_{l=j}^{k-1} e^{T}(l)\left(I \otimes V_{2}\right) e(l)+\sum_{j=k-\tau_{m}}^{k-1} \sum_{l=j}^{k-1} e^{T}(l)\left(I \otimes V_{3}\right) e(l)
\end{aligned}
$$




$$
+\sum_{j=k-\tau_{M}}^{k-1} \sum_{l=j}^{k-1} e^{T}(l)\left(I \otimes V_{4}\right) e(l) .
$$

Then, taking mathematical expectation of the forward difference formula $\Delta \mathcal{V}(k)=\mathcal{V}(k+1)-\mathcal{V}(k)$ along the trajectories of the system (6), we have

$$
\begin{aligned}
\mathbb{E}\left[\Delta \mathcal{V}_{1}(k)\right] & =\mathbb{E}\left[\mathcal{V}_{1}(k+1)-\mathcal{V}_{1}(k)\right] \\
& =\mathbb{E}\left[e^{T}(k+1) \sum_{q=1}^{N} \Pi_{p q} P_{q} e(k+1)-e^{T}(k) P_{p} e(k)\right] \\
& =\mathbb{E}[\eta(k)+e(k)]^{T} \sum_{q=1}^{N} \Pi_{p q} P_{q}[\eta(k)+e(k)]-e^{T}(k) P_{p} e(k),
\end{aligned}
$$

where $\eta(k)=e(k+1)-e(k)$

$$
\begin{aligned}
& \mathbb{E}\left[\Delta \mathcal{V}_{2}(k)\right]=\mathbb{E}\left[\mathcal{V}_{2}(k+1)-\mathcal{V}_{2}(k)\right] \\
& =\mathbb{E}\left[e^{T}(k) \sum_{j=1}^{6}\left(I \otimes Q_{j}\right) e(k)-e^{T}\left(k-d_{m}\right)\left(I \otimes Q_{1}\right) e\left(k-d_{m}\right)-e^{T}\left(k-d_{0}\right)\left(I \otimes Q_{2}\right)\right. \\
& e\left(k-d_{0}\right)-e^{T}\left(k-d_{M}\right)\left(I \otimes Q_{3}\right) e\left(k-d_{M}\right)-e^{T}\left(k-\tau_{m}\right)\left(I \otimes Q_{4}\right) e\left(k-\tau_{m}\right) \\
& \left.-e^{T}\left(k-\tau_{0}\right)\left(I \otimes Q_{5}\right) e\left(k-\tau_{0}\right)-e^{T}\left(k-\tau_{M}\right)\left(I \otimes Q_{6}\right) e\left(k-\tau_{M}\right)\right], \\
& \mathbb{E}\left[\Delta \mathcal{V}_{3}(k)\right]=E\left[\mathcal{V}_{3}(k+1)-\mathcal{V}_{3}(k)\right] \\
& \leq \mathbb{E}\left[\left(d_{0}-d_{m}+1\right) e^{T}(k)\left(I \otimes T_{1}\right) e(k)-e^{T}\left(k-d_{1}(k)\right)\left(I \otimes T_{1}\right) e\left(k-d_{1}(k)\right)\right. \\
& \left.+\left(\tau_{0}-\tau_{m}+1\right) e^{T}(k)\left(I \otimes T_{2}\right) e(k)-e^{T}\left(k-\tau_{1}(k)\right)\left(I \otimes T_{2}\right) e\left(k-\tau_{1}(k)\right)\right], \\
& \mathbb{E}\left[\Delta \mathcal{V}_{4}(k)\right]=\mathbb{E}\left[\mathcal{V}_{4}(k+1)-\mathcal{V}_{4}(k)\right] \\
& \leq \mathbb{E}\left[\left(d_{M}-d_{0}+1\right) e^{T}(k)\left(I \otimes U_{1}\right) e(k)-e^{T}\left(k-d_{2}(k)\right)\left(I \otimes U_{1}\right) e\left(k-d_{2}(k)\right)\right. \\
& \left.+\left(\tau_{M}-\tau_{0}+1\right) e^{T}(k)\left(I \otimes U_{2}\right) e(k)-e^{T}\left(k-\tau_{2}(k)\right)\left(I \otimes U_{2}\right) e\left(k-\tau_{2}(k)\right)\right], \\
& \mathbb{E}\left[\Delta \mathcal{V}_{5}(k)\right]=\mathbb{E}\left[\mathcal{V}_{5}(k+1)-\mathcal{V}_{5}(k)\right] \\
& \leq d_{m} e^{T}(k)\left(I \otimes V_{1}\right) e(k)-\frac{1}{d_{m}} \sum_{j=k-d_{m}}^{k-1} e^{T}\left(k\left(I \otimes V_{1}\right) \sum_{k-d_{m}}^{k-1} e(k)-\frac{1}{d_{M}} \sum_{j=k-d_{M}}^{k-1} e^{T}(k)\right. \\
& \times\left(I \otimes V_{2}\right) \sum_{j=k-d_{M}}^{k-1} e(k)+d_{M} e^{T}(k)\left(I \otimes V_{3}\right) e(k)+\tau_{m} e^{T}(k)\left(I \otimes V_{3}\right) e(k)-\frac{1}{\tau_{m}} \\
& \times \sum_{k-\tau_{m}}^{k-1} e^{T}(k)\left(I \otimes V_{3}\right) \sum_{k-\tau_{m}}^{k-1} e(k)+\tau_{m} e^{T}(k)\left(I \otimes V_{4}\right) e(k)-\frac{1}{\tau_{M}} \sum_{j=k-\tau_{M}}^{k-1} e^{T}(k)\left(I \otimes V_{4}\right) \\
& \times \sum_{j=k-\tau_{M}}^{k-1} e(k)
\end{aligned}
$$


Furthermore, we have

$$
\begin{aligned}
\eta(k)= & e(k+1)-e(k) \\
= & \left(\bar{A}+\bar{R} \mathbb{G} K_{p}+\bar{D}-1\right) e(k)+\bar{B} F(k, e(k))+\beta_{0} \bar{C} F\left(k, e\left(k-d_{1}(k)\right)\right)+\left(1-\beta_{0}\right) \bar{C} \\
& \times F\left(k, e\left(k-d_{2}(k)\right)\right)+\left(\beta(k)-\beta_{0}\right)\left[\bar{C} F\left(k, e\left(k-d_{1}(k)\right)\right)-\bar{C} F\left(k, e\left(k-d_{2}(k)\right)\right)\right] \\
& +\alpha_{0} \bar{G} e\left(k-\tau_{1}(k)\right)+\left(1-\alpha_{0}\right) \bar{G}\left(e\left(k-\tau_{2}(k)\right)\right)+\left(\alpha(k)-\alpha_{0}\right)\left[\bar{G}\left(e\left(k-\tau_{1}(k)\right)\right)\right. \\
& \left.-\bar{G}\left(e\left(k-\tau_{2}(k)\right)\right)\right]+w(k) .
\end{aligned}
$$

If we take $\mathcal{Z}_{p}=\left[\alpha_{1}\left(I \otimes P_{p}\right)^{T} \quad \alpha_{2}\left(I \otimes P_{p}\right)^{T}\right]^{T}$ with $\alpha_{i}>0(i=1,2)$, the following equation holds:

$$
\begin{aligned}
& 2 E\left\{\chi ^ { T } ( k ) \mathcal { Z } _ { p } \left[\left(\bar{A}+\bar{R} \mathbb{G} K_{p}+\bar{D}-1\right) e(k)+\bar{B} F(k, e(k))+\beta_{0} \bar{C} F\left(k, e\left(k-d_{1}(k)\right)\right)+\left(1-\beta_{0}\right) \bar{C}\right.\right. \\
& F\left(k, e\left(k-d_{2}(k)\right)\right)+\left(\beta(k)-\beta_{0}\right)\left[\bar{C} F\left(k, e\left(k-d_{1}(k)\right)\right)-\bar{C} F\left(k, e\left(k-d_{2}(k)\right)\right)\right]+\alpha_{0} \bar{G} e\left(k-\tau_{1}(k)\right) \\
& \left.\left.+\left(1-\alpha_{0}\right) \bar{G}\left(e\left(k-\tau_{2}(k)\right)\right)+\left(\alpha(k)-\alpha_{0}\right)\left[\bar{G}\left(e\left(k-\tau_{1}(k)\right)\right)-\bar{G}\left(e\left(k-\tau_{2}(k)\right)\right)\right]+w(k)-\eta(k)\right]\right\}=0,
\end{aligned}
$$

where $\chi^{T}(k)=\left[e^{T}(k) \quad \eta^{T}(k)\right]$.

From Assumption 3, we can obtain the following inequalities

$$
e^{T}(k)(I \otimes W)(I \otimes W)^{T} e(k)-F^{T}(k, x(k)) F(k, x(k)) \geq 0,
$$

$$
e^{T}\left(k-d_{1}(k)\right)(I \otimes E)(I \otimes E)^{T} e\left(k-d_{1}(k)\right)-F^{T}\left(k, x\left(k-d_{1}(k)\right)\right) F\left(k, x\left(k-d_{1}(k)\right)\right) \geq 0,
$$

$$
e^{T}\left(k-d_{2}(k)\right)(I \otimes H)(I \otimes H)^{T} e\left(k-d_{2}(k)\right)-F^{T}\left(k, x\left(k-d_{2}(k)\right)\right) F\left(k, x\left(k-d_{2}(k)\right)\right) \geq 0 .
$$

Combining (9) - (18), we have

$$
\mathbb{E}\left[\Delta V(k)-(\mu-1) V(k)-w^{T}(k) \bar{W} w(k)\right] \leq \zeta^{T}(k)\left\{\Omega_{[20 \times 20]}+\mathbb{E}_{1}^{T} \mathbb{E}_{1}+\mathbb{E}_{2}^{T} \mathbb{E}\right\} \zeta(k),
$$

where

$$
\begin{aligned}
& \zeta(k)=\left[\begin{array}{lllllll}
e^{T}(k) & e^{T}\left(k-d_{m}\right) & e^{T}\left(k-d_{0}\right) & e^{T}\left(k-d_{M}\right) & e^{T}\left(k-d_{1}(k)\right) & e^{T}\left(k-d_{2}(k)\right) & e^{T}\left(k-\tau_{m}\right)
\end{array}\right. \\
& \begin{array}{llllll}
e^{T}\left(k-\tau_{0}\right) & e^{T}\left(k-\tau_{M}\right) & e^{T}\left(k-\tau_{1}(k)\right) & e^{T}\left(k-\tau_{2}(k)\right) & F^{T}(k, e(k)) & F^{T}\left(k, e\left(k-d_{1}(k)\right)\right)
\end{array} \\
& \left.F^{T}\left(k, e\left(k-d_{2}(k)\right)\right) \quad \sum_{k-d_{m}}^{k-1} e^{T}(k) \quad \sum_{k-d_{M}}^{k-1} e^{T}(k) \quad \sum_{k-\tau_{m}}^{k-1} e^{T}(k) \sum_{k-\tau_{M}}^{k-1} e^{T}(k) \quad \eta^{T}(k) \quad w^{T}(k)\right], \\
& \mathbb{E}_{1}^{T}=[(I \otimes W)^{T}, 0,0,0,(I \otimes E)^{T},(I \otimes H)^{T}, \underbrace{0, \cdots, 0}_{14}],
\end{aligned}
$$


$\mathbb{E}_{2}^{T}=[\sqrt{\sum_{q=1}^{N} \Pi_{p q}\left(I \otimes P_{q}\right)}, \underbrace{0, \cdots, 0}_{17}, \sqrt{\sum_{q=1}^{N} \Pi_{p q}\left(I \otimes P_{q}\right)}, 0]^{T}$.

Further, by using schur complement to the right hand side of (19), we get the required LMI in (7). If the matrix inequality in (7) holds, it is obvious that $\mathbb{E}\left[\Delta V(k)-(\mu-1) V(k)-w^{T}(k) \bar{W} w(k)\right] \leq 0$.

$$
\begin{aligned}
\mathbb{E}\{\mathcal{V}(k+1)-\mathcal{V}(k)\} & \leq(\mu-1) \mathbb{E} \mathcal{V}(k)+E\left\{w^{T}(k) \bar{W} w(k)\right\} \\
& <(\mu-1) \mathbb{E} \mathcal{V}(k)+\lambda_{W} E\left\{w^{T}(k) w(k)\right\}
\end{aligned}
$$

where $\lambda_{W}=\lambda_{\max }(\bar{W})$. Thus, we can get $\mathbb{E}\{\mathcal{V}(k+1)\}<\mu \mathbb{E}\{\mathcal{V}(k)\}+\lambda_{W} \mathbb{E}\left\{w^{T}(k) w(k)\right\}$.

Further, if $\mu \geq 1$, it follows that

$$
\begin{aligned}
\mathbb{E}\{\mathcal{V}(k)\} & \leq \mu^{k} \mathbb{E}\{\mathcal{V}(0)\}+\lambda_{W} \mathbb{E}\left\{\sum_{n=0}^{k-1} \mu^{k-n-1} w^{T}(k) w(k)\right\} \\
& \leq \mu^{k} \mathbb{E} \mathcal{V}(0)+\mu^{k} \lambda_{W} \delta
\end{aligned}
$$

Next,we define the following parameters:

$$
\begin{aligned}
& \mathscr{P}_{p}=S_{p}^{-\frac{1}{2}} P_{p} S_{p}^{-\frac{1}{2}}, \mathscr{Q}_{b p}=S_{p}^{-\frac{1}{2}} Q_{b} S_{p}^{-\frac{1}{2}}, b=1, \cdots, 6, \mathscr{T}_{c p}=S_{p}^{-\frac{1}{2}} T_{c} S_{p}^{-\frac{1}{2}}, c=1,2, \\
& \mathscr{U}_{j p}=S_{p}^{-\frac{1}{2}} U_{j} S_{p}^{-\frac{1}{2}}, j=1,2, \mathscr{V}_{l p}=S_{p}^{-\frac{1}{2}} V_{l} S_{p}^{-\frac{1}{2}}, l=1,2,3,4 .
\end{aligned}
$$

Then, from (8), we can have

$$
\begin{aligned}
\mathbb{E}\{\mathcal{V}(0)\} & =\mathbb{E}\left\{e^{T}(0)\left(I \otimes S_{p}^{\frac{1}{2}} \mathscr{P}_{p} S_{p}^{\frac{1}{2}}\right) e(0)\right\}+\sum_{j=-d_{m}}^{-1} e^{T}(j)\left(I \otimes S_{p}^{\frac{1}{2}} \mathscr{Q}_{1 p} S_{p}^{\frac{1}{2}}\right) e(j)+\sum_{j=-d_{0}}^{-1} e^{T}(j) \\
& \times\left(I \otimes S_{p}^{\frac{1}{2}} \mathscr{Q}_{2 p} S_{p}^{\frac{1}{2}}\right) e(j)+\sum_{j=-d_{M}}^{-1} e^{T}(j)\left(I \otimes S_{p}^{\frac{1}{2}} \mathscr{Q}_{3 p} S_{p}^{\frac{1}{2}}\right) e(j)+\sum_{j=-\tau_{m}}^{-1} e^{T}(j)\left(I \otimes S_{p}^{\frac{1}{2}} \mathscr{Q}_{4 p} S_{p}^{\frac{1}{2}}\right) \\
& \times e(j)+\sum_{j=-\tau_{0}}^{-1} e^{T}(j)\left(I \otimes S_{p}^{\frac{1}{2}} \mathscr{Q}_{5 p} S_{p}^{\frac{1}{2}}\right) e(j)+\sum_{j=-\tau_{M}}^{-1} e^{T}(j)\left(I \otimes S_{p}^{\frac{1}{2}} \mathscr{Q}_{6 p} S_{p}^{\frac{1}{2}}\right) e(j)+\sum_{j=k-d_{1}(0)}^{-1} e^{T}(j) \\
& \times\left(I \otimes S_{p}^{\frac{1}{2}} \mathscr{T}_{1 p} S_{p}^{\frac{1}{2}}\right) e(j)+\sum_{j=d_{m}}^{d_{0}-1} \sum_{l=-j}^{-1} e^{T}(l)\left(I \otimes S_{p}^{\frac{1}{2}} \mathscr{T}_{1 p} S_{p}^{\frac{1}{2}}\right) e(l)+\sum_{j=-\tau_{1}(0)}^{-1} e^{T}(j)\left(I \otimes S_{p}^{\frac{1}{2}} \mathscr{T}_{2 p} S_{p}^{\frac{1}{2}}\right) \\
& \times e(j)+\sum_{j=\tau_{m}}^{\tau_{0}-1} \sum_{l=-j}^{-1} e^{T}(l)\left(I \otimes S_{p}^{\frac{1}{2}} \mathscr{T}_{2 p} S_{p}^{\frac{1}{2}}\right) e(l)+\sum_{j=-d_{2}(0)}^{-1} e^{T}(j)\left(I \otimes S_{p}^{\frac{1}{2}} \mathscr{U}_{1 p} S_{p}^{\frac{1}{2}}\right) e(j)+\sum_{j=d_{0}+1}^{d_{m}-1} \sum_{l=-j}^{-1} \\
& \times e^{T}(l)\left(I \otimes S_{p}^{\frac{1}{2}} \mathscr{U}_{1 p} S_{p}^{\frac{1}{2}}\right) e(l)+\sum_{s=k-\tau_{2}(0)}^{-1} e^{T}(j)\left(I \otimes S_{p}^{\frac{1}{2}} \mathscr{U}_{2 p} S_{p}^{\frac{1}{2}}\right) e(j)+\sum_{j=\tau_{0}+1}^{\tau_{m}-1} \sum_{l=-j}^{-1} e^{T}(l) \\
& \times\left(I \otimes S_{p}^{\frac{1}{2}} \mathscr{U}_{2 p} S_{p}^{\frac{1}{2}}\right) e(l)+\sum_{j=-d_{m}}^{-1} \sum_{l=j}^{-1} e^{T}(l)\left(I \otimes S_{p}^{\frac{1}{2}} \mathscr{V}_{1 p} S_{p}^{\frac{1}{2}}\right) e(l)+\sum_{j=-d_{M}}^{-1} \sum_{l=j}^{k-1} e^{T}(l)\left(I \otimes S_{p}^{\frac{1}{2}} \mathscr{V}_{2 p} S_{p}^{\frac{1}{2}}\right) e(l)
\end{aligned}
$$




$$
\begin{aligned}
& \left.+\sum_{j=-\tau_{m}}^{-1} \sum_{l=j}^{-1} e^{T}(l)\left(I \otimes S_{p}^{\frac{1}{2}} \mathscr{V}_{3 p} S_{p}^{\frac{1}{2}}\right) e(l)+\sum_{j=-\tau_{M}}^{-1} \sum_{l=j}^{k-1} e^{T}(l)\left(I \otimes S_{p}^{\frac{1}{2}} \mathscr{V}_{4 p} S_{p}^{\frac{1}{2}}\right) e(l)\right\} \\
& \leq c_{1}\left[\lambda_{2}+\lambda_{3} d_{m}+\lambda_{4} d_{0}+\lambda_{5} d_{M}+\lambda_{6} \tau_{m}+\lambda_{7} \tau_{0}+\lambda_{8} \tau_{M}+\lambda_{9} d_{0}+\lambda_{9} \frac{\left(d_{0}-d_{m}\right)\left(d_{m}+d_{0}-1\right)}{2}\right. \\
& +\lambda_{10} \tau_{0}+\lambda_{10} \frac{\left(\tau_{0}-\tau_{m}\right)\left(\tau_{m}+\tau_{0}-1\right)}{2}+\lambda_{11} d_{M}+\lambda_{11} \frac{\left(d_{0}+d_{m}\right)\left(d_{m}-d_{0}+1\right)}{2}+\lambda_{12} \tau_{M} \\
& +\lambda_{12} \frac{\left(\tau_{0}+\tau_{m}\right)\left(\tau_{m}-\tau_{0}+1\right)}{2}+\lambda_{13} \frac{d_{m}\left(d_{m}-1\right)}{2}+\lambda_{14} \frac{d_{M}\left(d_{M}-1\right)}{2}+\lambda_{15} \frac{\tau_{m}\left(\tau_{m}-1\right)}{2} \\
& \left.+\lambda_{16} \frac{\tau_{M}\left(\tau_{M}-1\right)}{2}\right] \\
& \leq \zeta_{1} c_{1} .
\end{aligned}
$$

Hence $\mathbb{E}\{\mathcal{V}(k)\} \geq \lambda_{1} \mathbb{E}\left\{e^{T}(k)\left(I \otimes S_{p}\right) e(k)\right\}$.

By using the inequalities (20) and (21), we can obtain

$$
\begin{aligned}
\mathbb{E}\left\{e^{T}(k)\left(I \otimes S_{p}\right) e(k)\right\} & \leq \frac{1}{\lambda_{1}}\left(\zeta_{1} c_{1}+\lambda_{W} \delta\right) \mu^{k} \\
& <c_{2}, \quad \forall k=1,2, \cdots, N
\end{aligned}
$$

From the above computations, we can conclude that the closed-loop error system 6 is stochastically finite-time bounded with respect to $\left(c_{1}, c_{2}, S_{p}, \mathbb{N}, \delta\right)$.

\section{B. Stochastic finite-time dissipative}

A sufficient condition is given to ensure the finite-time boundedness with dissipative performance of CDNs have analyzed in the following theorem

Theorem 2. Under assumptions 1 and 2 , for given scalar $\mu \geq 1$, the error system (6) is stochastic finite-time dissipative with respect to $\left(c_{1}, c_{2}, S_{p}, \mathbb{N}, \delta, \mathbb{L}, \mathbb{M}, \mathbb{R}\right)$, if there exist symmetric matrices $X_{p}>0(p \in \psi), \hat{Q}_{b p}>0(b=1,2, \cdots, 6), \hat{T}_{c p}>0, \hat{U}_{c p}>0 \quad(c=1,2), \hat{V}_{l p}>0,(l=1,2,3,4)$ such that the following conditions hold:

$$
\begin{gathered}
\hat{\Psi}=\left[\begin{array}{cccc}
\hat{\Gamma}_{20 \times 20} & \hat{\Xi}_{1} & \hat{\Xi}_{2} & \Upsilon \\
* & -I & 0 & 0 \\
* & * & \hat{\Xi}_{3} & 0 \\
* & * & * & -I
\end{array}\right]<0 \\
\hat{\zeta}_{1} c_{1}+\lambda_{\mathbb{R}} \delta<\lambda_{1}^{-1} c_{2} \mu^{-k}
\end{gathered}
$$


where $\hat{\Gamma}_{1,1}=-\mu\left(I \otimes X_{p}\right)+\left(I \otimes \hat{Q}_{1 p}\right)+\left(I \otimes \hat{Q}_{2 p}\right)+\left(I \otimes \hat{Q}_{3 p}\right)+\left(I \otimes \hat{Q}_{4 p}\right)+\left(I \otimes \hat{Q}_{5 p}\right)+$ $+\left(I \otimes \hat{Q}_{6 p}\right)+\left(d_{0}-d_{m}+1\right)\left(I \otimes \hat{T}_{1 p}\right)+\left(\tau_{0}-\tau_{m}+1\right)\left(I \otimes \hat{T}_{2 p}\right)+\left(d_{M}-d_{0}+1\right)\left(I \otimes \hat{U}_{1 p}\right)+$ $\left(\tau_{M}-\tau_{0}+1\right)\left(I \otimes \hat{U}_{2 p}\right)+d_{m}\left(I \otimes \hat{V}_{1 p}\right)+d_{M}\left(I \otimes \hat{V}_{3 p}\right)+\tau_{m}\left(I \otimes \hat{V}_{3 p}\right)+\tau_{M}\left(I \otimes \hat{V}_{4 p}\right)+\kappa_{1} \bar{A}(I \otimes$ $\left.X_{p}\right)+\kappa_{1}\left(I \otimes X_{p}\right)^{T} \bar{A}^{T}+\kappa_{1} \bar{R} \mathbb{G} Y_{p}+\kappa_{1} Y_{p}^{T} \mathbb{G}^{T} \bar{R}^{T}+\kappa_{1} \bar{D}\left(I \otimes X_{p}\right)+\kappa_{1}\left(I \otimes X_{p}\right)^{T} \bar{D}^{T}-\kappa_{1}(I \otimes$ $\left.X_{p}\right)-\kappa_{1}\left(I \otimes X_{p}\right)^{T}, \quad \hat{\Gamma}_{1,10}=\alpha_{0} \kappa_{1} \bar{G}, \quad \hat{\Gamma}_{1,11}=\kappa_{1}\left(1-\alpha_{0}\right) \bar{G}, \hat{\Gamma}_{1,12}=\kappa_{1} \bar{B}, \hat{\Gamma}_{1,13}=\kappa_{1} \beta_{0} \bar{C}$, $\hat{\Gamma}_{1,14}=\kappa_{1}\left(1-\beta_{0}\right) \bar{C}, \hat{\Gamma}_{1,19}=-\kappa_{1}\left(I \otimes X_{p}\right)+\kappa_{2}\left(I \otimes X_{p}\right) \bar{A}+\kappa_{2} \bar{R} \mathbb{G} Y_{p}+\kappa_{2}\left(I \otimes X_{p}\right) \bar{D}-$ $\kappa_{2}\left(I \otimes X_{p}\right), \quad \hat{\Gamma}_{1,20}=\kappa_{1}-\left(I \otimes X_{p}\right) \bar{J}^{T} \mathbb{M}, \hat{\Gamma}_{2,2}=-\left(I \otimes \hat{Q}_{1 p}\right), \hat{\Gamma}_{3,3}=-\left(I \otimes \hat{Q}_{2 p}\right), \hat{\Gamma}_{4,4}=$ $-\left(I \otimes \hat{Q}_{3 p}\right), \hat{\Gamma}_{5,5}=-\left(I \otimes \hat{T}_{1 p}, \hat{\Gamma}_{6,6}=-\left(I \otimes \hat{U}_{1 p}\right), \hat{\Gamma}_{7,7}=-\left(I \otimes \hat{Q}_{4 p}\right), \hat{\Gamma}_{8,8}=-\left(I \otimes \hat{Q}_{5 p}\right)\right.$, $\hat{\Gamma}_{9,9}=-\left(I \otimes \hat{Q}_{6 p}\right), \hat{\Gamma}_{10,10}=-\left(I \otimes \hat{T}_{2 p}\right), \Gamma_{10,19}=\alpha_{0} \kappa_{2} \bar{G}^{T}\left(I \otimes X_{p}\right), \hat{\Gamma}_{11,11}=-\left(I \otimes \hat{U}_{2 p}\right)$, $\hat{\Gamma}_{11,19}=\left(1-\alpha_{0}\right) \kappa_{2} \bar{G}^{T}\left(I \otimes X_{p}\right), \hat{\Gamma}_{12,12}=-I, \hat{\Gamma}_{12,19}=\kappa_{2} \bar{B}^{T}, \hat{\Gamma}_{13,13}=-I, \hat{\Gamma}_{13,19}=\kappa_{2} \beta_{0} \bar{C}^{T}$, $\hat{\Gamma}_{14,14}=-I, \hat{\Gamma}_{14,19}=\kappa_{2}\left(1-\beta_{0}\right) \bar{C}^{T}, \hat{\Gamma}_{15,15}=-1 / d_{m}\left(I \otimes \hat{V}_{1 p}\right), \hat{\Gamma}_{16,16}=-1 / d_{M}\left(I \otimes \hat{V}_{2 p}\right), \hat{\Gamma}_{17,17}=$ $-1 / \tau_{m}\left(I \otimes \hat{V}_{3 p}\right), \hat{\Gamma}_{18,18}=-1 / \tau_{M}\left(I \otimes \hat{V}_{4 p}\right), \hat{\Gamma}_{19,19}=-\kappa_{2}\left(I \otimes X_{p}\right)-\kappa_{2}\left(I \otimes X_{p}\right)^{T}, \hat{\Gamma}_{19,20}=\kappa_{2}$, $\hat{\Gamma}_{20,20}=-\mathbb{R}+\gamma I, \hat{\Xi}_{1}=[\left(I \otimes X_{p}\right)(I \otimes W), 0,0,0,0,\left(I \otimes X_{p}\right)(I \otimes E),\left(I \otimes X_{p}\right)(I \otimes H), \underbrace{0 \cdots 0}_{13}]$ $\hat{\Xi}_{2}=\left[\hat{\Xi}_{21} \hat{\Xi}_{22} \cdots \hat{\Xi}_{2 N}\right], \hat{\Xi}_{21}=[\sqrt{\Pi}_{p 1}\left(I \otimes X_{p}\right) \underbrace{0 \cdots 0}_{17}, \sqrt{\Pi}_{p 1}\left(I \otimes X_{p}\right), 0]^{T}, \hat{\Xi}_{22}=\left[\sqrt{\Pi}_{p 2}(I \otimes\right.$ $\left.X_{p}\right) \underbrace{0 \cdots 0}_{17}, \sqrt{\Pi}_{p 2}\left(I \otimes X_{p}\right), 0]^{T}, \cdots, \hat{\Xi}_{2 N}=[\sqrt{\Pi}_{p N}\left(I \otimes X_{p}\right) \underbrace{0 \cdots 0}_{17}, \sqrt{\Pi}_{p N}\left(I \otimes X_{p}\right), 0]^{T}, \hat{\Xi}_{3}=$ $-\operatorname{diag}\left\{\left(I \otimes X_{1}\right),\left(I \otimes X_{2}\right), \cdots,\left(I \otimes X_{N}\right)\right\}, \Upsilon=[\bar{J}^{T} \sqrt{-\bar{L}}, \underbrace{0 \cdots 0}_{19}], \hat{\zeta}_{1}=\lambda_{2}^{-1}+\lambda_{1}^{-2} \lambda_{3} d_{m}+$ $\lambda_{1}^{-2} \lambda_{4} d_{0}+\lambda_{1}^{-2} \lambda_{5} d_{M}+\lambda_{1}^{-2} \lambda_{6} \tau_{m}+\lambda_{1}^{-2} \lambda_{7} \tau_{0}+\lambda_{1}^{-2} \lambda_{8} \tau_{M}+\lambda_{1}^{-2} \lambda_{9} d_{0}+\lambda_{1}^{-2} \lambda_{9} \frac{\left(d_{0}-d_{m}\right)\left(d_{m}+d_{0}-1\right)}{2}+$ $\lambda_{1}^{-2} \lambda_{10} \tau_{0}+\lambda_{1}^{-2} \lambda_{10} \frac{\left(\tau_{0}-\tau_{m}\right)\left(\tau_{m}+\tau_{0}-1\right)}{2}+\lambda_{1}^{-2} \lambda_{11} d_{M}+\lambda_{1}^{-2} \lambda_{11} \frac{\left(d_{0}+d_{m}\right)\left(d_{m}-d_{0}+1\right)}{2}+\lambda_{1}^{-2} \lambda_{12} \tau_{M}+\lambda_{1}^{-2} \lambda_{12}$ $\times \frac{\left(\tau_{0}+\tau_{m}\right)\left(\tau_{m}-\tau_{0}+1\right)}{2}+\lambda_{1}^{-2} \lambda_{13} \frac{d_{m}\left(d_{m}-1\right)}{2}+\lambda_{1}^{-2} \lambda_{14} \frac{d_{M}\left(d_{M}-1\right)}{2}+\lambda_{1}^{-2} \lambda_{15} \frac{\tau_{m}\left(\tau_{m}-1\right)}{2}+\lambda_{1}^{-2} \lambda_{16} \frac{\tau_{M}\left(\tau_{M}-1\right)}{2}$. Moreover, the gain matrices are given by $K_{p}=Y_{p} X_{p}^{-1}$.

Proof: In order to describe the dissipative performance of the closed-loop system (6), we consider the energy function $\mathcal{J}$ as

$$
\mathcal{J}=\mathbb{E}\left\{\sum_{k=0}^{\mathcal{N}}\left[e^{T}(k) \mathcal{L} e(k)+2 e^{T}(k) \mathcal{M} w(k)+w^{T}(k)[\mathcal{R}-\gamma I] w(k)\right]\right\} .
$$

The proof follows from Theorem 1, it follows that $\mathbb{E}\{\Delta \mathcal{V}(k)-(\mu-1) V(k)-\mathcal{J}\} \leq 0$.

Thus, $\mathbb{E}\{\Delta \mathcal{V}(k)\}<\mathbb{E}\left\{\mu \mathcal{V}(k)+e^{T}(k) \mathcal{L} e(k)+2 e^{T}(k) \mathcal{M} w(k)+w^{T}(k)[\mathcal{R}-\gamma I] w(k)\right\}$.

By simple modifications, it is quite easy to get that

$$
\begin{aligned}
\mathbb{E}\{\Delta V(k)\} \leq & \mathbb{E}\left\{\mu^{k} V(0)+\sum_{t=0}^{k-1} \mu^{k-t-1} e^{T}(k) \mathcal{L} e(k)+2 \sum_{t=0}^{k-1} \mu^{k-t-1} e^{T}(k) \mathcal{M} w(k)\right. \\
& \left.+\sum_{t=0}^{k-1} \mu^{k-t-1} w^{T}(k)[\mathcal{R}-\gamma I] w(k)\right\} .
\end{aligned}
$$


Under zero initial condition and the fact $V(k) \geq 0, \forall k=1,2, \cdots, \mathcal{N}$, we have

$$
\mathbb{E}\left\{\sum_{t=0}^{k-1} \mu^{k-t-1} e^{T}(k) \mathcal{L} e(k)+2 \sum_{t=0}^{k-1} \mu^{k-t-1} e^{T}(k) \mathcal{M} w(k)+\sum_{t=0}^{k-1} \mu^{k-t-1} w^{T}(k)[\mathcal{R}-\gamma I] w(k)\right\} \geq 0 .
$$

Further, if $\mu \geq 1$, the above inequality implies that

$$
\mathbb{E}\left\{\sum_{k=0}^{\mathcal{N}} e^{T}(k) \mathcal{L} e(k)+2 \sum_{k=0}^{\mathcal{N}} e^{T}(k) \mathcal{M} w(k)+\sum_{k=0}^{\mathcal{N}} w^{T}(k)[\mathcal{R}-\gamma I] w(k)\right\} \geq 0 .
$$

Then, from (25), it is easily to get the inequality in the Definition 1 . To complete the proof of this theorem, let $X_{p}=P_{p}^{-1}, \hat{Q_{b p}}=X_{p} Q_{p} X_{p}(b=1,2, \cdots, 6), \hat{T_{c p}}=X_{p} T_{c} X_{p}, \hat{U_{c p}}=X_{p} U_{c} X_{p}(c=$ $1,2), \hat{V}_{l p}=X_{p} V_{l} X_{p}(l=1,2,3,4)$, then performing the congruence transformations to (7) by $\operatorname{diag}\{\underbrace{\left(I \otimes X_{p}\right), \cdots,\left(I \otimes X_{p}\right)}_{11},(I \otimes I),(I \otimes I),(I \otimes I), \underbrace{\left(I \otimes X_{p}\right), \cdots,\left(I \otimes X_{p}\right)}_{5},(I \otimes I),(I \otimes I), \mathbb{U}\}$, where $\mathbb{U}=-\operatorname{diag}\left\{\left(I \otimes X_{1}\right),\left(I \otimes X_{2}\right), \cdots,\left(I \otimes X_{N}\right)\right\}$ and Letting $K_{p} X_{p}=Y_{p}$, we can easily to get the required LMI 22,.

Further, $\lambda_{1} \leq\left(I \otimes S_{p}^{-\frac{1}{2}} P_{p} S_{p}^{-\frac{1}{2}}\right) \leq \lambda_{2}, 0<\left(I \otimes S_{p}^{-\frac{1}{2}} Q_{1} S_{p}^{-\frac{1}{2}}\right)<\lambda_{3}, 0<\left(I \otimes S_{p}^{-\frac{1}{2}} Q_{2} S_{p}^{-\frac{1}{2}}\right)<\lambda_{4}$, $0<\left(I \otimes S_{p}^{-\frac{1}{2}} Q_{3} S_{p}^{-\frac{1}{2}}\right)<\lambda_{5}, \quad 0<\left(I \otimes S_{p}^{-\frac{1}{2}} Q_{4} S_{p}^{-\frac{1}{2}}\right)<\lambda_{6}, 0<\left(I \otimes S_{p}^{-\frac{1}{2}} Q_{5} S_{p}^{-\frac{1}{2}}\right)<\lambda_{7}$, $0<\left(I \otimes S_{p}^{-\frac{1}{2}} Q_{6} S_{p}^{-\frac{1}{2}}\right)<\lambda_{8}, 0<\left(I \otimes S_{p}^{-\frac{1}{2}} T_{1} S_{p}^{-\frac{1}{2}}\right)<\lambda_{9}, 0<\left(I \otimes S_{p}^{-\frac{1}{2}} T_{2} S_{p}^{-\frac{1}{2}}\right)<\lambda_{10}$, $0<\left(I \otimes S_{p}^{-\frac{1}{2}} U_{1} S_{p}^{-\frac{1}{2}}\right)<\lambda_{11}, 0<\left(I \otimes S_{p}^{-\frac{1}{2}} U_{2} S_{p}^{-\frac{1}{2}}\right)<\lambda_{12}, 0<\left(I \otimes S_{p}^{-\frac{1}{2}} V_{1} S_{p}^{-\frac{1}{2}}\right)<\lambda_{13}$, $0<\left(I \otimes S_{p}^{-\frac{1}{2}} V_{2} S_{p}^{-\frac{1}{2}}\right)<\lambda_{14}, 0<\left(I \otimes S_{p}^{-\frac{1}{2}} V_{3} S_{p}^{-\frac{1}{2}}\right)<\lambda_{15}$, and $0<\left(I \otimes S_{p}^{-\frac{1}{2}} V_{4} S_{p}^{-\frac{1}{2}}\right)<\lambda_{16}$.

Then, according to the congruence transformations, these above relations can be altered into $\lambda_{2}^{-1}(I \otimes$ $\left.S_{p}^{-1}\right)<\left(I \otimes X_{p}\right)<\lambda_{1}^{-1}\left(I \otimes S_{p}^{-1}\right), \quad 0<\left(I \otimes \hat{Q}_{1 p}\right)<\lambda_{1}^{-2} \lambda_{3}\left(I \otimes S_{p}^{-1}\right), \quad 0<\left(I \otimes \hat{Q}_{2 p}\right)<$ $\lambda_{1}^{-2} \lambda_{4}\left(I \otimes S_{p}^{-1}\right), \quad 0<\left(I \otimes \hat{Q}_{3 p}\right)<\lambda_{1}^{-2} \lambda_{5}\left(I \otimes S_{p}^{-1}\right), \quad 0<\left(I \otimes \hat{Q}_{4 p}\right)<\lambda_{1}^{-2} \lambda_{6}\left(I \otimes S_{p}^{-1}\right)$, $0<\left(I \otimes \hat{Q}_{5 p}\right)<\lambda_{1}^{-2} \lambda_{7}\left(I \otimes S_{p}^{-1}\right), 0<\left(I \otimes \hat{Q}_{6 p}\right)<\lambda_{1}^{-2} \lambda_{8}\left(I \otimes S_{p}^{-1}\right), \quad 0<\left(I \otimes \hat{T}_{1 p}\right)<$ $\lambda_{1}^{-2} \lambda_{9}\left(I \otimes S_{p}^{-1}\right), \quad 0<\left(I \otimes \hat{T}_{2 p}\right)<\lambda_{1}^{-2} \lambda_{10}\left(I \otimes S_{p}^{-1}\right), \quad 0<\left(I \otimes \hat{U}_{1 p}\right)<\lambda_{1}^{-2} \lambda_{11}\left(I \otimes S_{p}^{-1}\right)$, $0<\left(I \otimes \hat{U}_{2 p}\right)<\lambda_{1}^{-2} \lambda_{12}\left(I \otimes S_{p}^{-1}\right), \quad 0<\left(I \otimes \hat{V}_{1 p}\right)<\lambda_{1}^{-2} \lambda_{13}\left(I \otimes S_{p}^{-1}\right), \quad 0<\left(I \otimes \hat{V}_{2 p}\right)<$ $\lambda_{1}^{-2} \lambda_{14}\left(I \otimes S_{p}^{-1}\right), 0<\left(I \otimes \hat{V}_{3 p}\right)<\lambda_{1}^{-2} \lambda_{15}\left(I \otimes S_{p}^{-1}\right), 0<\left(I \otimes \hat{V}_{4 p}\right)<\lambda_{1}^{-2} \lambda_{16}\left(I \otimes S_{p}^{-1}\right)$, it can easily received the inequality (23). Hence, completes the proof.

Remark 2. By using Definitions 2 and 3, it should be noted that if $w(k)=0$, the concept of SFTB is reduced to SFTS. Also we can observe that if the error system (6) is stochastically finite time stable then the proposed CDNs (1) is finite time synchronized under the control law (5). In CDNs (1), removing the nonlinear function and external disturbance and considering only one mode in operation, then it can be rewritten as follows:

$x_{l}(k+1)=A x_{l}(k)+B f\left(k, x_{l}(k)\right)+C f\left(k, x_{l}(k)\right)+\sum_{m=1}^{N} d_{l m} \Gamma_{1} x_{m}(k)+\sum_{m=1}^{N} g_{l m} \Gamma_{2} x_{m}(k-\tau(k))$ 


$$
\begin{aligned}
& +R V_{l}^{f}(k), \\
z_{l}(k)= & J x_{l}(k), \quad l=1,2, \ldots, N .
\end{aligned}
$$

Then the closed-loop error system (6) becomes

$$
\begin{aligned}
e(k+1)= & \bar{A} e(k)+\bar{B} F(k, e(k))+\beta_{0} \bar{C} F(k, e(k))+\bar{D} e(k)+\alpha_{0} \bar{G} e\left(k-\tau_{1}(k)\right)+\left(1-\alpha_{0}\right) \\
& \times \bar{G}\left(e\left(k-\tau_{2}(k)\right)\right)+\left(\alpha(k)-\alpha_{0}\right)\left[\bar{G}\left(e\left(k-\tau_{1}(k)\right)\right)-\bar{G}\left(e\left(k-\tau_{2}(k)\right)\right)\right]+\bar{R} \mathbb{G} K e(k), \\
z(k)= & \bar{J} e(k) .
\end{aligned}
$$

Corollary 1. For given matrices $S>0$, and scalars $\mu \geq 1, c_{1}>0, c_{2}>0, \mathbb{N}$ and $\alpha_{i} \geq 0(i=1,2)$ and under the Assumptions 1 and 2, the error system (27) is stochastic finite-time synchronization with respect to $\left(c_{1}, c_{2}, S, \mathbb{N}\right)$, if there exist symmetric matrices $X>0, \hat{Q}_{b}>0(b=1,2,3), \hat{T}_{c}>0$, $\hat{U}_{c}>0(c=1,2), \hat{V}_{l}>0(l=1,2)$ such that the following inequalities hold:

$$
\begin{gathered}
\hat{\Psi}=\left[\begin{array}{cc}
\hat{\Gamma}_{10 \times 10} & \hat{\Xi}_{1} \\
* & -I
\end{array}\right]<0, \\
\hat{\zeta}_{1} c_{1}<\lambda_{1}^{-1} c_{2} \mu^{-k},
\end{gathered}
$$

where $\hat{\Gamma}_{1,1}=-\mu(I \otimes X)+\left(I \otimes \hat{Q}_{1}\right)+\left(I \otimes \hat{Q}_{2}\right)+\left(I \otimes \hat{Q}_{3}\right)+\left(\tau_{0}-\tau_{m}+1\right)\left(I \otimes \hat{T}_{1}\right)+\left(\tau_{M}-\tau_{0}+1\right)(I \otimes$ $\left.\hat{U}_{1}\right)+\tau_{m}\left(I \otimes \hat{V}_{1}\right)+\tau_{M}\left(I \otimes \hat{V}_{2}\right)+\kappa_{1} \bar{A}(I \otimes X)+\kappa_{1}(I \otimes X)^{T} \bar{A}^{T}+\kappa_{1} \bar{R} \mathbb{G} Y+\kappa_{1} Y^{T} \mathbb{G}^{T} \bar{R}^{T}+\kappa_{1} \bar{D}(I \otimes$ $X)+\kappa_{1}(I \otimes X)^{T} \bar{D}^{T}-\kappa_{1}(I \otimes X)-\kappa_{1}(I \otimes X)^{T}, \quad \hat{\Gamma}_{1,5}=\alpha_{0} \kappa_{1} \bar{G}, \hat{\Gamma}_{1,6}=\kappa_{1}\left(1-\alpha_{0}\right) \bar{G}, \hat{\Gamma}_{1,7}=\kappa_{1} \bar{B}$, $\hat{\Gamma}_{1,10}=-\kappa_{1}(I \otimes X)+\kappa_{2}(I \otimes X) \bar{A}+\kappa_{2} \bar{R} \mathbb{G} Y+\kappa_{2}(I \otimes X) \bar{D}-\kappa_{2}(I \otimes X), \hat{\Gamma}_{2,2}=-\left(I \otimes \hat{Q}_{1}\right)$, $\hat{\Gamma}_{3,3}=-\left(I \otimes \hat{Q}_{2}\right), \quad \hat{\Gamma}_{4,4}=-\left(I \otimes \hat{Q}_{3}\right), \hat{\Gamma}_{5,5}=-\left(I \otimes \hat{T}_{1}\right), \Gamma_{5,10}=\alpha_{0} \kappa_{2} \bar{G}^{T}(I \otimes X), \quad \hat{\Gamma}_{6,6}=$ $-\left(I \otimes \hat{U}_{1}\right), \hat{\Gamma}_{6,10}=\left(1-\alpha_{0}\right) \kappa_{2} \bar{G}^{T}(I \otimes X), \hat{\Gamma}_{7,7}=-I, \hat{\Gamma}_{7,10}=\kappa_{2} \bar{B}^{T}, \hat{\Gamma}_{8,8}=-1 / \tau_{m}\left(I \otimes \hat{V}_{1}\right)$, $\hat{\Gamma}_{9,9}=-1 / \tau_{M}\left(I \otimes \hat{V}_{2}\right), \hat{\Gamma}_{10,10}=-\kappa_{2}(I \otimes X)-\kappa_{2}(I \otimes X)^{T}, \hat{\Xi}_{1}=[(I \otimes X)(I \otimes W), \underbrace{0 \cdots 0}_{9}]$ and remaining parameters are zero. Moreover, the gain matrices are estimated by $K=Y X^{-1}$.

Proof: Consider the following LKF

$$
\begin{aligned}
& \mathcal{V}_{1}(k)=e^{T}(k)(I \otimes P) e(k), \\
& \mathcal{V}_{2}(k)=\sum_{j=k-\tau_{m}}^{k-1} e^{T}(j)\left(I \otimes Q_{1}\right) e(j)+\sum_{j=k-\tau_{0}}^{k-1} e^{T}(j)\left(I \otimes Q_{2}\right) e(s)+\sum_{j=k-\tau_{M}}^{k-1} e^{T}(j)\left(I \otimes Q_{3}\right) e(j), \\
& \mathcal{V}_{\ni}(k)=\sum_{j=k-\tau_{1}(k)}^{k-1} e^{T}(j)\left(I \otimes T_{1}\right) e(j)+\sum_{j=\tau_{m}}^{\tau_{0}-1} \sum_{l=k-j}^{k-1} e^{T}(l)\left(I \otimes T_{1}\right) e(l), \\
& \mathcal{V}_{4}(k)=\sum_{s=k-\tau_{2}(k)}^{k-1} e^{T}(j)\left(I \otimes U_{1}\right) e(j)+\sum_{j=\tau_{0}+1}^{\tau_{m}-1} \sum_{l=k-j}^{k-1} e^{T}(l)\left(I \otimes U_{1}\right) e(l),
\end{aligned}
$$




$$
\begin{aligned}
& \mathcal{V}_{5}(k)=\sum_{j=k-\tau_{m}}^{k-1} \sum_{l=j}^{k-1} e^{T}(l)\left(I \otimes V_{1}\right) e(l)+\sum_{j=k-\tau_{M}}^{k-1} \sum_{l=j}^{k-1} e^{T}(l)\left(I \otimes V_{2}\right) e(l),
\end{aligned}
$$

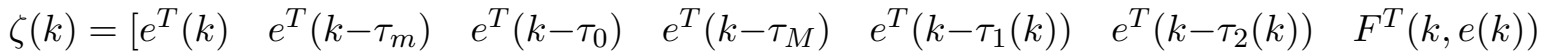

$$
\begin{aligned}
& \left.\sum_{k-\tau_{m}}^{k-1} e^{T}(k) \sum_{k-\tau_{M}}^{k-1} e^{T}(k) \quad \eta^{T}(k)\right]
\end{aligned}
$$

Remaining proof follows from Theorem 2, we can obtain the required result. Thus, the proof is completed.

\section{Numerical Simulations}

This section provides a simulation examples to show the effectiveness and superiority of the established criteria for finite-time synchronization of the proposed CDNs.

Example 1. Consider a class of CDNs in the form of (2) with three nodes and dimension of the state vector of each node is two.

Mode 1:

$$
\begin{aligned}
& A_{1}=\left[\begin{array}{cc}
-1.43 & 1.14 \\
1.23 & -1.26
\end{array}\right], \quad B_{1}=\left[\begin{array}{cc}
-0.25 & 2.0095 \\
0.15 & -1.45
\end{array}\right], \quad C_{1}=\left[\begin{array}{cc}
0.54 & 0.05 \\
0.42 & 0.23
\end{array}\right], \quad J_{1}=\left[\begin{array}{cc}
1.01 & 0.02 \\
0 & 0.01
\end{array}\right], \\
& R_{1}=\left[\begin{array}{cc}
1.021 & 0 \\
3.86 & 0.011
\end{array}\right]
\end{aligned}
$$

Mode 2:

$$
\begin{aligned}
& A_{2}=\left[\begin{array}{cc}
-0.79 & 0.26 \\
0.31 & 0.13
\end{array}\right], \quad B_{2}=\left[\begin{array}{cc}
1.05 & 1.95 \\
-0.05 & 2.05
\end{array}\right], \quad C_{2}=0.1 *\left[\begin{array}{cc}
-2.14 & 0.05 \\
0.042 & -1.23
\end{array}\right], \quad J_{2}=\left[\begin{array}{cc}
1.1 & 0.01 \\
0 & 1.2
\end{array}\right], \\
& R_{2}=\left[\begin{array}{cc}
0.052 & 1.96 \\
0.01 & -0.1
\end{array}\right] \\
& \alpha_{0}=0.6, \beta_{0}=0.4 .
\end{aligned}
$$

The inner and outer coupling matrices of non-delayed and delayed terms are taken as

$\Gamma_{1}=\operatorname{diag}\{0.09,0.09\}, \Gamma_{2}=\operatorname{diag}\{0.25,0.25\}$ and $D_{1}=D_{2}=G_{1}=G_{2}=\left[\begin{array}{ccc}-0.1 & 0.1 & 0 \\ 0.2 & -0.6 & 0.4 \\ 0 & 0.2 & -0.2\end{array}\right]$.

The non-linear function $f\left(x_{i}(k)\right)=\left[\begin{array}{l}\tanh \left(0.2 x_{i 1}(k)\right) \\ \tanh \left(0.2 x_{i 2}(k)\right)\end{array}\right]$, it can easily satisfies Assumption 3 with the matrices $\mathscr{K}_{1}=\mathscr{K}_{2}=\mathscr{K}_{3}=\operatorname{diag}\{0.4,0.4\}$ and also the noise signal is chosen as $w(k)=0.05 \exp (-0.1 k) \sin k$.

Further, the time-delays are taken as $d_{1}(k)=3.01+0.25 \sin (0.1 k), d_{2}(k)=3.5+0.25 \sin (0.1 k)$, $\tau_{1}(k)=1.05+0.25 \sin (0.5 k), \tau_{2}(k)=2.01+0.25 \sin (0.02 k)$ and the actuator failure matrix 
is $\mathbb{G}=\operatorname{diag}\{0.8,0.8\}$. The transition probability matrices are $\left[\begin{array}{cc}0.2 & 0.8 \\ 0.35 & 0.65\end{array}\right]$ and $\left[\begin{array}{cc}0.6 & 0.4 \\ 0.3 & 0.7\end{array}\right]$. Moreover,the other parameters are considered as $c_{1}=0.01, c_{2}=13.8344, S_{1}=S_{2}=0.2$, $\mathbb{N}=20, \delta=0.1, \gamma=0.4729, \mu=1.1$. Also we choose $\mathbb{L}=-1.15, \mathbb{M}=\operatorname{diag}\{1,1\}$ and $\mathbb{R}=\operatorname{diag}\{1.2,1.2\}$.

Now by using the aforementioned parameters and applying MATLAB toolbox to solve the LMIs formulated in Theorem 2, the following feedback control gain matrices are estimated

$$
\begin{aligned}
& K_{11}=\left[\begin{array}{cc}
0.5860 & 3.3082 \\
-32.0133 & 16.1103
\end{array}\right], \quad K_{12}=\left[\begin{array}{cc}
0.5860 & 3.3082 \\
-32.0133 & 16.1103
\end{array}\right], \quad K_{13}=\left[\begin{array}{cc}
0.5860 & 3.3082 \\
-32.0133 & 16.1103
\end{array}\right], \\
& K_{21}=\left[\begin{array}{cc}
174.2709 & -106.9181 \\
-3.3945 & 2.1245
\end{array}\right], \quad K_{22}=\left[\begin{array}{cc}
174.2709 & -106.9181 \\
-3.3945 & 2.1245
\end{array}\right], \quad K_{23}=\left[\begin{array}{cc}
174.2709 & -106.9181 \\
-3.3945 & 2.1245
\end{array}\right],
\end{aligned}
$$

For the simulation purposes, the initial conditions for the states of the nodes and the isolated node are taken as $x_{1}(0)=\left[\begin{array}{ll}4 & 2\end{array}\right]^{T}, x_{2}(0)=\left[\begin{array}{ll}-1 & 1\end{array}\right]^{T}, x_{3}(0)=\left[\begin{array}{ll}2 & -4\end{array}\right]^{T}$ and $s_{1}(0)=\left[\begin{array}{ll}1 & 2\end{array}\right]^{T}$.

Based on the above values, simulation results are presented in Figs. 1,7. Specifically, the state responses of the first, second and third nodes together with the isolated node are plotted in Fig.1. It can be seen from this figure that the states of the nodes are exactly synchronized with the isolated node. The error responses with and without control are depicted in Fig 2. It can be observed from Fig 2 (b) that the error response without control cannot reach zero within a finite period. Figs 3 and 4 represent simulation of semi-Markov jumping process and disturbance signal, respectively. In addition, the Bernoulli random variable $\alpha(k)$ with $\alpha_{0}=0.6$ and $\beta(k)$ with $\beta_{0}=0.4$ are plotted in Figs.5 and 6. The time history of $e_{i}^{T} S_{p} e_{i}(k)(i=1, \cdots 6)$ is depicted in Fig 7. From these figures, it can be realized that the $\mathrm{CDNs}(2)$ is finite-time bounded with respect to $(0.01,13.8344,0.2,20,0.1)$ even in the existence of the network induced imperfections such as actuator fault and time-varying delays.

Example 2. Consider the discrete-time system 27) with parameters as follows:

$$
A=2 *\left[\begin{array}{cc}
0.041 & -0.004 \\
-1.001 & 0.001
\end{array}\right], \quad B=1.5 *\left[\begin{array}{cc}
0.0084 & 0 \\
0 & 0.0001
\end{array}\right], \quad C=\left[\begin{array}{ll}
0 & 0 \\
0 & 0
\end{array}\right], \quad R=\left[\begin{array}{cc}
0.4106 & 0.984 \\
0.9456 & 0.6766
\end{array}\right] \text {. }
$$

The inner and outer coupling matrices of non-delayed and delayed terms are taken as $\Gamma_{1}=\operatorname{diag}\{0.09,0.09\}$, $\Gamma_{2}=\operatorname{diag}\{0.25,0.25\}$ and $D_{1}=G_{1}=\left[\begin{array}{ccc}-0.4 & 0.4 & 0 \\ 0.1 & -0.1 & 0 \\ 0 & 0 & 0\end{array}\right]$.

The non-linear function $f\left(x_{i}(k)\right)=\left[\begin{array}{l}\tanh \left(0.2 x_{i 1}(k)\right) \\ \tanh \left(0.2 x_{i 2}(k)\right)\end{array}\right]$, it can easily satisfies Assumption 3 with the matrices $\mathscr{K}_{1}=\operatorname{diag}\{0.1,0.1\}$. 
Further, the time-delays are taken as $\tau_{1}(k)=1.25+0.25 \sin (0.5 k), \tau_{2}(k)=2.5+0.25 \sin (0.02 k)$ and the actuator failure matrix is $\mathbb{G}=\operatorname{diag}\{0.8,0.8\}$. Moreover, the other parameters are considered as $c_{1}=1.5, c_{2}=9.5379, S=0.1, \mathbb{N}=20, \gamma=0.4729, \mu=1.1$.

Now, by using the aforementioned parameters and applying MATLAB toolbox to solve the LMIs formulated in Corollary 1, the following feedback control gain matrices are estimated

$K_{11}=K_{12}=K_{13}=\left[\begin{array}{cc}3.5056 & -0.6347 \\ -1.3886 & 0.4554\end{array}\right]$.

For the simulation purposes, the initial conditions for the states of the nodes and the isolated node are taken as $x_{1}(0)=\left[\begin{array}{ll}3 & 2\end{array}\right]^{T}, x_{2}(0)=\left[\begin{array}{ll}-1 & 1\end{array}\right]^{T}, x_{3}(0)=\left[\begin{array}{ll}1 & 2\end{array}\right]^{T}$ and $s_{1}(0)=\left[\begin{array}{ll}1 & -4\end{array}\right]^{T}$.

Based on the initial condition and system parameter values, simulation results are presented in Figs. 8[10. Specifically, the state responses of the first, second and third nodes together with the isolated node are plotted in Fig 8 . It can be seen from this figure that the states of the nodes are exactly synchronized with the isolated node. The error responses with control are depicted in Fig 9 and the time history of $e_{i}^{T} S e_{i}(k)(i=1, \cdots 6)$ is depicted in Fig 10 . From these figures, it can be realized that the CDNs 27) is finite-time synchronization with respect to $(1.5,9.5379,0.1,20)$ even in the existence of the network induced imperfections such as actuator fault.
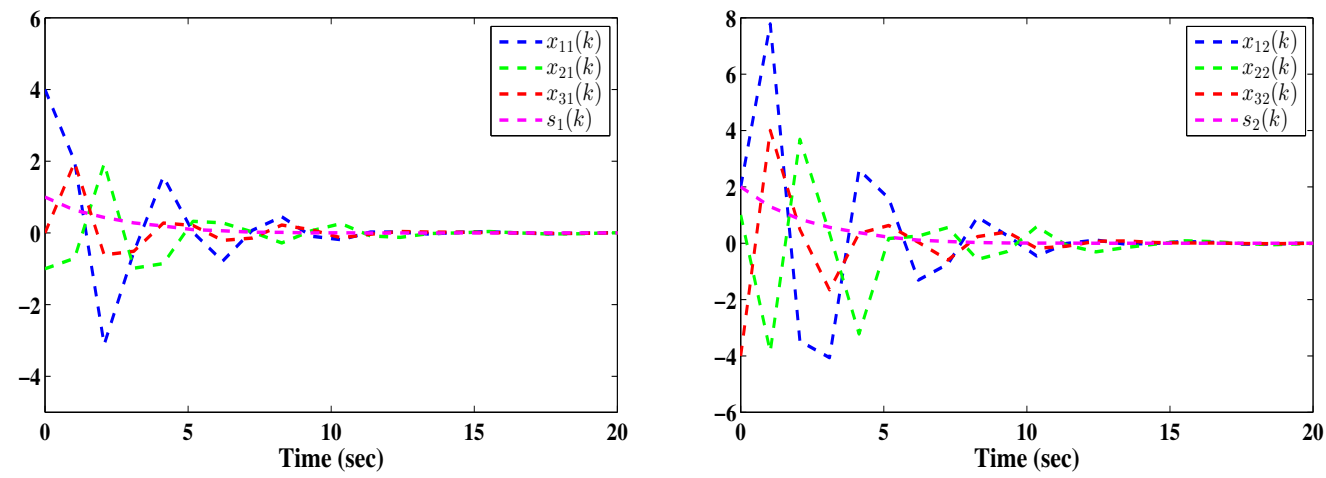

Figure 1: State responses of the system (1)

\section{Conclusion}

In this paper, the dissipative based finite-time synchronization problem has been investigated for a discrete-time CDNs subject to semi-Markovian jumping parameters, probabilistic time-varying delays and actuator faults through the reliable control. By constructing suitable Lyapunov functional method and Kronecker product properties, the required criteria for ensuring the finite-time synchronization with dissipativity performance index of the CDNs have been obtained in the form of LMIs. 


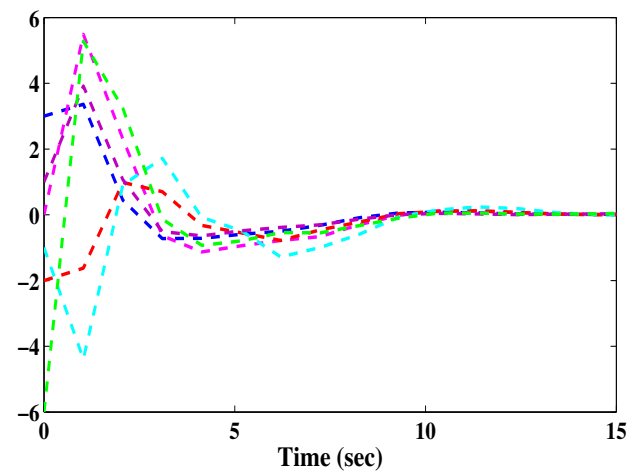

(a) with control

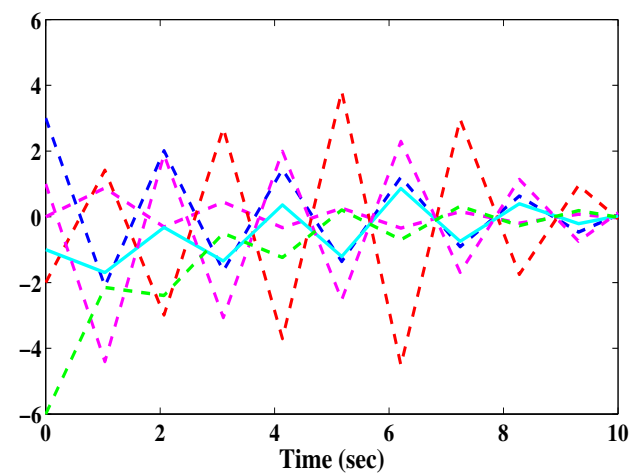

(b) without control

Figure 2: State responses of the error system (6) with and without control
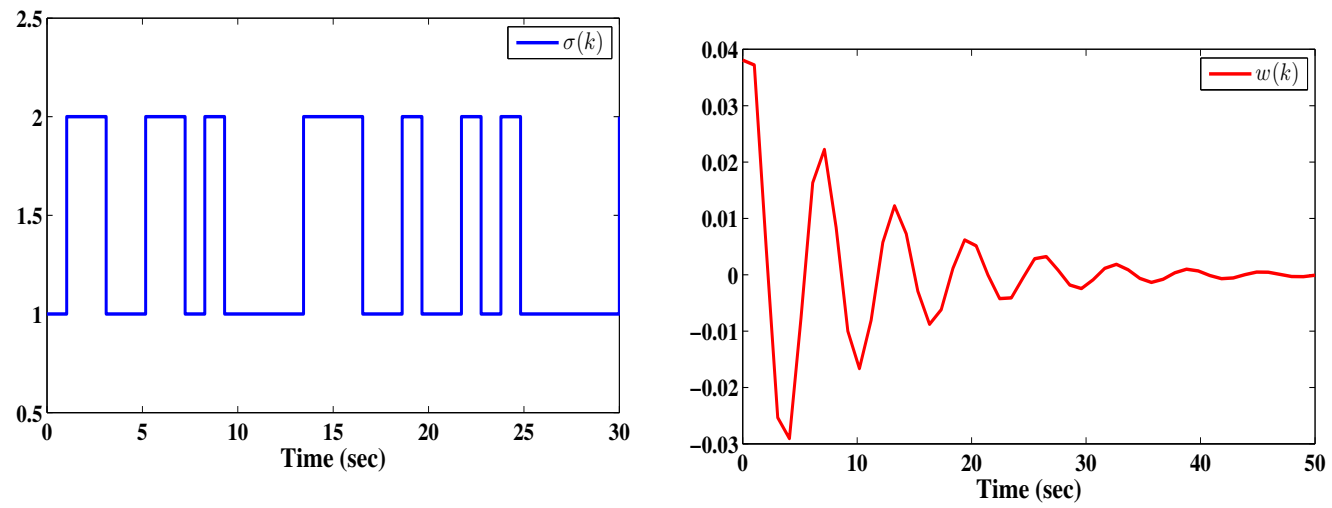

Figure 3: Semi-Markovian switching signal $\sigma(k)$

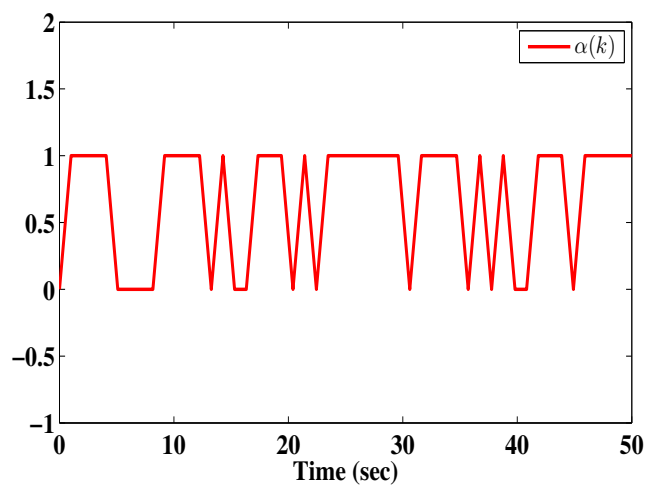

Figure 5: Stochastic parameter $\alpha(k)$ with $\alpha_{0}=0.6$

Figure 4: Disturbance signal

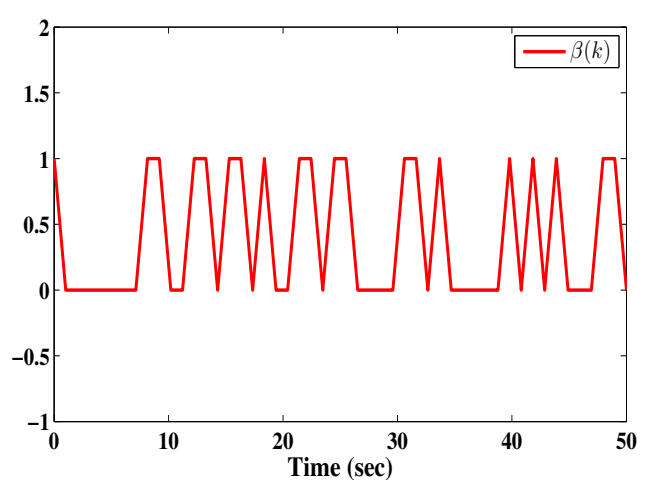

Figure 6: Stochastic parameter $\beta(k)$ with $\beta_{0}=0.4$ 


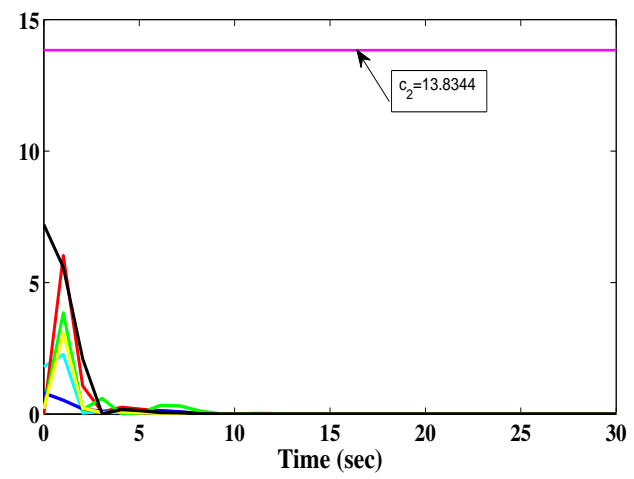

Figure 7: Evolution of $e_{i}^{T}(k) S_{p} e_{i}(k)$
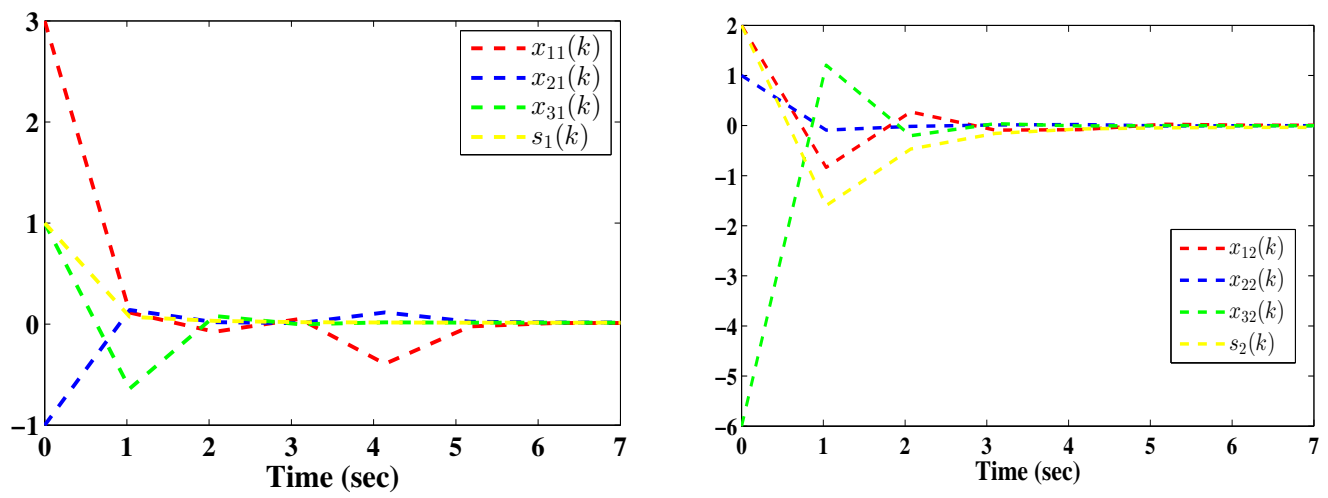

Figure 8: Response of system states

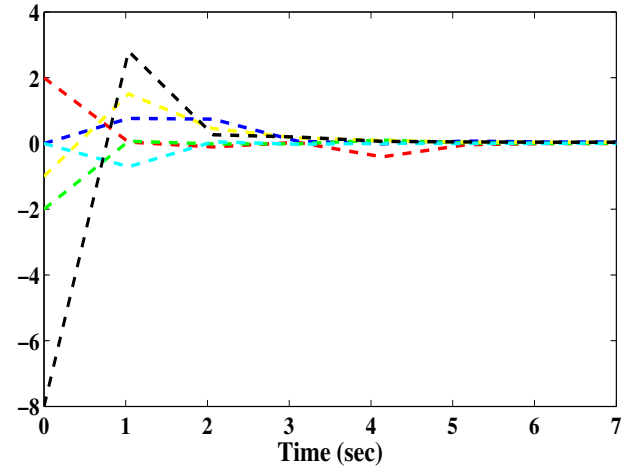

Figure 9: Error response of CDNs 25]

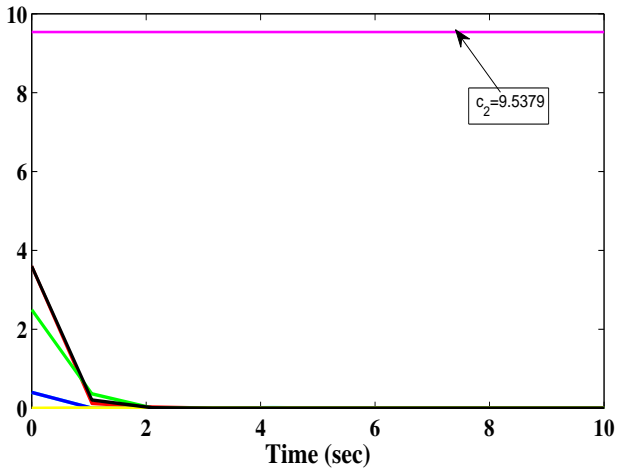

Figure 10: Evolution of $e_{i}^{T}(k) S e_{i}(k)$

Finally, two numerical examples have been exploited to show the effectiveness of the established proposed results. 


\section{Conflict of interest}

The authors declare that they have no conflict of interest.

\section{Ethical approval}

This article does not contain any studies with human participants performed by any of the authors.

\section{Acknowledgement}

This work was financially supported by the UGC-BSR Research Start-Up-Grant under Ministry of Human Resource Development, Government of India (Grant No. F.30-410/2018(BSR)). The work of Yong-Ki Ma was supported by the National Research Foundation of Korea (NRF) grant funded by the Korea government (MSIT) (No. 2021R1F1A1048937)

\section{References}

[1] H. Zhang, J. Hu, H. Liu, X. Yu and F. Liu, Recursive state estimation for time-varying complex networks subject to missing measurements and stochastic inner coupling under random access protocol, Neurocomputing, 346 (2019) 48-57.

[2] H. Dong, N. Hou, Z. Wang and W. Ren, Variance-Constrained state estimation for complex networks with randomly varying topologies, IEEE transactions on neural networks and learning systems, 29 (2017) 2757 - 2768.

[3] W. Li, Y. Jia and J. Du, Recursive state estimation for complex networks with random coupling strength, Neurocomputing, 219 (2017) 1-8.

[4] Y. Hao, J. Han, Y. Lin and L. Liu, Vulnerability of complex networks under three-level-tree attacks, Physica A: Statistical mechanics and its applications, 462 (2016) 674-683.

[5] G. He, J. Fang, W. Zhang and Z. Li, Synchronization of switched complex dynamical networks with non-synchronized sub networks and stochastic disturbances, Neurocomputing, 171 (2016) $39-47$. 
[6] Z. Li, J. Fang, T. Huang and Q. Miao, Synchronization of stochastic discrete-time complex networks with partial mixed impulse effects, Journal of the Franklin Institute, 354 (2017) 41964214.

[7] B. Li, Z. Wang and L. Ma, An Event-triggered pinning control approach to synchronization of discrete-time stochastic complex dynamical networks, IEEE Transactions on neural networks and learning systems, 29 (2018) 5812 - 5822.

[8] F. Han, G. Wei, D. Ding and Y. Song, Finite-horizon bounded $H_{\infty}$ synchronization and state estimation for discrete-time complex networks:local performance analysis, IET Control Theory and Applications, 11 (2017) 827-837.

[9] S. Sheng, X. Zhang and G. Lu, Finite-time outer synchronization for complex networks with Markov jump topology via hyprid control and its application to image encryption, Journal of the Franklin Institute, 355 (2018) 6493-6519.

[10] Q. Li, J. Guo, C. Sun, Y. Wu and Z. Ding, Finite-Time Synchronization for a Class of Dynamical Complex Networks with Nonidentical Nodes and Uncertain Disturbance, Journal of Systems Science and Complexity, 32 (2019) 818-834.

[11] H. Shen, Ju H. Park and Z.G. Wu, Finite-time reliable $L_{2}-L_{\infty} / H_{\infty}$ control for Takagi-Sugeno fuzzy systems with actuator faults, IET Control Theory and Applications, 8 (2014) 688-696.

[12] R. Sakthivel, S. Santra, K. Mathiyalagan and S. Marshal Anthoni, Robust reliable sampled-data control for offshore steel jacket platforms with nonlinear perturbations, Nonlinear Dynamics, 78 (2014) 1109-1123.

[13] H. Dong, Z. Wang, S.X. Ding and H. Gao, Finite-horizon reliable control with randomly occurring uncertainties and nonlinearities subject to output quantization, Automatica, 52 (2015b) $355-362$.

[14] R. Sakthivel, A. Arunkumar, K. Mathiyalagan and S. Selvi, Robust reliable control for uncertain vehicle suspension systems with input delays, Journal of Dynamic Systems, Measurement and Control, 137 (2015) doi:10.1115/1.4028776.

[15] Z. Qunjiao, W. Xiaoqun and L. Jie, Pinning synchronization of discrete-time complex netwoks with different time-varying delays, Journal of systems science and complexity, 32 (2019) 15601571. 
[16] Q. Zhang, G. Chen and L. Wan, Exponential synchronization of discrete-time impulsive dynamical networks with time-varying delays and stochastic disturbances, Neurocomputing, 309 (2018) 62-69.

[17] M.J. Park, O.M. Kwon, Stability and stabilization of discrete-time T-S fuzzy systems with timevarying delay via Cauchy-Schwartz-based summation inequality, IEEE Transactions on fuzzy systems, 25 (2017) 128-140.

[18] X. Yang, D.W.C. Ho, J. Lu and Q. Song, Finite-time cluster synchronization of T-S fuzzy complex networks with discontinuous subsystems and random coupling delays, IEEE Transactions on Fuzzy Systems, 23 (2015) 2302-2316.

[19] R. Cheng and M. Peng, Adaptive synchronization for complex networks with probabilistic timevarying delays, Journal of the Franklin Institute, 353 (2016) 5099-5120.

[20] R. Cheng, M. Peng, J. Yu and H. Li, Synchronization for discrete-time complex networks with probabilistic time delays, Physica A:Statistical Mechanics and its applications, 525 (2019) 10881101.

[21] A. Wang, T. Dong, and X. Liao, Event-triggered synchronization strategy for complex dynamical networks with the Markovian switching topologies, Neural Networks, 74 (2016) 52-57.

[22] H. Ren, F. Deng, Y. Peng, Finite time synchronization of Markovian jumping stochastic complex dynamical systems with mix delays via hybrid control strategy, Neurocomputing 272 (2018) 683-693.

[23] Nasim Akbari, Ali Sadr and Ali Kazemy, Exponential Synchronization of Markovian Jump Complex Dynamical Networks with Uncertain Transition Rates and Mode-Dependent Coupling Delay, Circuits, Systems, and Signal Processing, 39 (2020) 3875-3906.

[24] H. Shen, J.H. Park,Z.-G. Wu and Z. Zhang, Finite-time $H_{\infty}$ synchronization for complex networks with semi-Markov jump topology, Communications in Nonlinear Science and Numerical Simulation, 24 (2015) 40-51.

[25] K. Liang, M. Dai, H. Shen, J. Wang, Z. Wang and B. Chen, $L 2-L_{\infty}$ synchronization for singularly perturbed complex networks with semi-Markov jump topology, Applied Mathematics and Computation, 321 (2018) 450-462.

[26] J.C. Willems, Dissipative dynamical systems part I: General theory, Archive for Rational Mechanics and Analysis, 45 (1972) 321-351. 
[27] H. Peng, R. Lu, Y. Xu and F. Yao, Dissipative non-fragile state estimation for Markovian complex networks with coupling transmission delays, Neurocomputing, 275 (2018) 1576-1584.

[28] L. Ma, X. Fang, Y. Yuan, J. Zhang and Y. Bo, Dissipative control for nonlinear Markovian jump systems with mixed time-delays: The discrete-time case, International Journal of Robust and Nonlinear Control, (2020) DOI: 10.1002/rnc.4906.

[29] J. Wang, X. Hu, Y. Wei and Z. Wang, Sampled-data synchronization of semi-Markov jump complex dynamical networks subject to generalized dissipativity property, Applied Mathematics and Computation, 346 (2019) 853-864.

[30] J. Cheng, H. Zhu, S. Zhong, Y. Zhang, Y. Li, Finite-time H control for a class of discrete-time Markovian jump systems with partly unknown time-varying transition probabilities subject to average dwell time switching, International Journal of Systems Science, 46 (2015) 1080-1093.

[31] Y. Zhang, P. Shi, S.K. Nguang and H.R. Karimi, Observer-based finite-time fuzzy H1 control for discrete-time systems with stochastic jumps and time-delays, Signal Processing, 97 (2014) 252-261.

[32] J. Song, Y. Niu, and S. Wang, Robust finite-time dissipative control subject to randomly occurring uncertainties and stochastic fading measurements, Journal of the Franklin Institute, 354 (2017) 3706-3723. 